\title{
Wavelet-based multiscale analysis of geomagnetic disturbance
}

\author{
N. Zaourar ${ }^{1}$, M. Hamoudi ${ }^{1}$, M. Mandea ${ }^{2}$, G. Balasis ${ }^{3}$, and M. Holschneider ${ }^{4}$ \\ ${ }^{1}$ Laboratoire de Géophysique, FSTGAT, B.P. 32 USTHB, 16123 Bab-Ezzouar, Alger, Algérie \\ ${ }^{2}$ Centre National d'Etudes Spatiales, 2 Place Maurice Quentin, 75039 Paris Cedex 01, France \\ ${ }^{3}$ Institute for Astronomy, Astrophysics, Space Applications and Remote Sensing, National Observatory of Athens, Metaxa and Vasileos Pavlou, \\ Penteli, 15236 Athens, Greece \\ ${ }^{4}$ Universität Potsdam, Institut für Mathematik, Postfach 601553 D-14415 Potsdam, Germany
}

(Received May 16, 2011; Revised April 30, 2013; Accepted May 2, 2013; Online published December 6, 2013)

\begin{abstract}
The dynamics of external contributions to the geomagnetic field is investigated by applying time-frequency methods to magnetic observatory data. Fractal models and multiscale analysis enable obtaining maximum quantitative information related to the short-term dynamics of the geomagnetic field activity. The stochastic properties of the horizontal component of the transient external field are determined by searching for scaling laws in the power spectra. The spectrum fits a power law with a scaling exponent $\beta$, a typical characteristic of self-affine time-series. Local variations in the power-law exponent are investigated by applying wavelet analysis to the same time-series. These analyses highlight the self-affine properties of geomagnetic perturbations and their persistence. Moreover, they show that the main phases of sudden storm disturbances are uniquely characterized by a scaling exponent varying between 1 and 3, possibly related to the energy contained in the external field. These new findings suggest the existence of a long-range dependence, the scaling exponent being an efficient indicator of geomagnetic activity and singularity detection. These results show that by using magnetogram regularity to reflect the magnetosphere activity, a theoretical analysis of the external geomagnetic field based on local power-law exponents is possible.
\end{abstract}

Key words: Geomagnetic field, magnetosphere, geomagnetic storm, multiscale analysis, spectral exponent.

\section{Introduction}

When the geomagnetic field interacts with the solar magnetic field carried by the solar wind (the interplanetary magnetic field), a considerable transfer of energy takes place into the Earth's magnetosphere. Magnetic effects can be seen via the ring current, dominating at middle and low latitudes, and a system of ionospheric electrojet currents at higher latitudes. Magnetic disturbance phenomena, such as magnetic storms (MSs) and substorms, are linked to these current systems (Kamide et al., 1998). The fieldaligned currents provide a link between the high- and the low-latitude current distributions, and contribute to increasing the complexity of the entire system. All these current systems affect the geomagnetic field, and especially its horizontal component at the Earth's surface.

The sudden and sharp changes in the geomagnetic field temporal variations, storms and substorms are two kinds of different events, as storms are a global reaction to extreme interplanetary conditions, mostly after solar eruptive events such as coronal mass ejections (CMEs). The most important geomagnetic storms are found to be mainly caused by CMEs (Gosling et al., 1990; Bothmer and Schwenn, 1995; Tsurutani and Gonzalez, 1995; Zhang et al., 2003). Storms and substorms are major constituents of geomagnetic activ-

Copyright (C) The Society of Geomagnetism and Earth, Planetary and Space Sciences (SGEPSS); The Seismological Society of Japan; The Volcanological Society of Japan; The Geodetic Society of Japan; The Japanese Society for Planetary Sciences; TERRAPUB.

doi:10.5047/eps.2013.05.001 ity and play a crucial role in efforts to define space weather (Kamide et al., 1998; Freeman, 2001; Angelopoulos et al., 2008). Intense geomagnetic storms occurring at extreme solar events, can affect modern life by disturbing technological systems (Baker, 2000; Daglis et al., 2001). The strength of geomagnetic storms is mainly reflected by the Dst index (http://swdcwww.kugi.kyoto-u.ac.jp/).

The internal mechanism of geomagnetic fluctuations accompanying the development of geomagnetic storms and substorms is a complex nonlinear system. Some previous works have demonstrated the statistical self-affinity properties observed in the geomagnetic times series (Chang, 1999; Sitnov et al., 2001; Uritsky et al., 2001, 2006; Kovacs et al., 2001; Lui, 2002; Wanliss, 2005a; Balasis et al., 2006; Wanliss and Dobias, 2007).

It has been found that variations of the external geomagnetic field exhibit a power-law spectrum with a spectral exponent varying over different lengths of time. This behaviour indicates that the external geomagnetic field is a multiscale process (Uritsky et al., 2001; Balasis et al., 2006). Considering current efforts in understanding the magnetic geo-environment and improving space weather forecasting, it is evident that the use of classical methods, such as the Fourier Transform for analysing the magnetic signal, or the geomagnetic index Dst, cannot yield any information on the temporal evolution of geomagnetic storm activity (Balasis et al., 2006). Therefore, new signal processing tools for the analysis and modelling of geomagnetic activity are required. 
The mathematical properties of the wavelet transform such as the "time-scale" representation are extensively used and show the intrinsic multiscale complexity of the geomagnetic signal. With a local decomposition of a multiscale signal, wavelet analysis is particularly suited to detect and follow the time evolution of the frequency distribution of a given time series (Ivanov et al., 2001; Kovacs et al., 2001; Lui, 2002; Mendes et al., 2005; Mandea and Balasis, 2006; Zaourar et al., 2006; Gaci et al., 2010). This property is particularly important when one considers non-stationary processes. Moreover, wavelet analysis is a powerful multiscale resolution technique, well suited to deeply understanding the complex features of real world processes: different 'kinds' of (multi)-fractality, long-range dependence (LRD), non-stationary, oscillatory behaviour and trends. Wavelet-based estimators have been used very successfully for estimating scaling behaviour applied to time series (Balasis et al., 2006; Zaourar et al., 2010). The central properties of a self-affine process enables a scaling relation to be established between wavelet coefficients and scale. The local spectral exponent is then easily derived.

Extending research into the fractal spectral properties of the SYM-H index, studied by Wanliss (2005a), and of the Dst index, developed by Balasis et al. (2006), we examine the geomagnetic disturbances recorded at three geomagnetic observatories. The purpose of this study is to apply wavelet analysis to reveal the fractal properties and LRD characteristics of geomagnetic disturbances, directly reflected by geomagnetic field recordings. Indeed, these local spectral features of the scale laws governing these signals highlight the self-affine properties of geomagnetic perturbations and their persistence.

This paper presents a description of the mathematical tools applied, and a detailed discussion of the results obtained. Section 2 presents the methods of analysis, starting with the scaling process and continuing with the continuous wavelet analysis of the scaling processes. An in-depth account of the data selection is given in Section 3, and the main results can be found in Section 4. Some possible directions for future research are briefly discussed in the concluding Section 5.

\section{Analysis Methods}

\subsection{Scaling process}

The notion of scale invariance is defined closely as the absence of characteristic scales of a time series. Its main consequence is that the whole and its parts cannot be statistically distinguished from each other. The concept of scaling behaviour is associated with different features such as LRD, self-affinity or multi-fractality. All these processes are characterized by the fact that their power spectra behave as a power law of the scale. The most popular model that displays scaling behaviour is certainly fractional Brownian motion (fBm). Introduced by Mandelbrot and Ness (1968), $\mathrm{fBm}$ is a family of Gaussian processes $\left\{B_{H}(t), t>0\right\}$ indexed by a single parameter $H$ called Hurst's parameter with a mean of 0 , and stationary increments and $B_{H}(0)=0$. Its covariance is given by:

$$
E\left\{B_{H}(t) B_{H}(u)\right\}=\frac{\sigma^{2}}{2}\left[t^{2 H}+|u|^{2 H}+|t-u|^{2 H}\right],
$$

where $0<H<1, \sigma>0$ and $E\{$.$\} denotes the expected$ value. Note that, with $H=1 / 2, B_{1 / 2}(t)$ corresponds to the classical Brownian motion with independent increments. One of the main assets of $\mathrm{fBm}$ is that it obeys the self-affine relationship:

$$
B_{H}(\lambda t) \approx \lambda^{H} B_{H}(t) .
$$

Here, the symbol $\approx$ means statistical equality, and $\lambda$ is real $(\lambda>0)$. Equation (2) expresses the fact that in selfaffine processes one must rescale the horizontal and vertical directions differently ( $\lambda$ for $t$ and $\lambda^{H}$ for $B_{H}(t)$ ) in order to have statistical invariance. Thus, self-affine processes are anisotropic by construction in the horizontal and vertical directions.

As with the fBm, the geomagnetic fluctuations are governed by scale laws where the power spectra are approximately inversely proportional to the frequency $f>0$ (Mandelbrot, 1985):

$$
S(f) \sim f^{-\beta},
$$

where the spectral exponent $\beta=2 H+1$, controls the degree of correlation between successive points. Following Pilkington and Todoeschuck (1990), two properties depend on the value of $\beta$.

(i) The degree of correlation: $\beta=0$ corresponds to the classical case of white noise where the values are completely uncorrelated and for which interpolation is forbidden. $\beta<0$, corresponds to anti-correlated values. $\beta>0$ is for positively-correlated values.

(ii) The stationarity of the series: for $\beta<1$, the sequence is weakly stationary in the sense that two separate samples have the same average, which can be defined as an average of the stochastic process. If $\beta>1$, the sequence is non-stationary (Pilkington and Todoeschuck, 1990, 1991), and the process is deflected from its initial value; therefore neither an average, nor a power spectrum, can be precisely defined. For $1<\beta<3$, the Gaussian scale noise, as defined by Pilkington and Todoeschuck (1990), is not stationary and corresponds to an $\mathrm{fBm}$. However, the power spectrum can be defined for all real $\beta$. In all cases, a self-affine time series with a non-zero $\beta$ has long-range (as well as short-range) persistence and antipersistence. For small $\beta$, correlations with large lag are small but are non-zero (Pilkington and Todoeschuck, 1990). This cannot conform with a time series which is not self-affine; such a series may have short-range persistence (either strong or weak).

It is important to point out that the fBms display scaling behaviour in a wide sense, i.e. all the features that we have mentioned above are present in the process. Currently, however, the scaling holds only within a finite range and is typically approximated. A broad class of signals corresponds to fractal processes, usually related to scaling in the limit of small scales. Such time series are described by a (local) scaling exponent, which is related to the degree of regularity of a signal. A sudden transition in the time series is a characteristic of a system that undertakes a phase transition, or achieves a new behaviour mode.

The fractal concept is usually used in a broader sense and refers to any process that shows some sort of self-affinity. The spectral range of the self-affine random of structure is not yet clear. The power spectrum is one of the most suit- 
able measures for discussing these time issues. When the Fourier power spectrum of a random process is proportional to a power of frequency, this process is self-affine (Turcotte, 1997; Kantelhardt et al., 2006; Kantelhardt, 2008).

2.2 The continuous wavelet analysis of scaling processes

The continuous wavelet transform (CWT) is a convolution product of the data sequence $s(t)$ with the scaled and translated version of the mother wavelet (basis function), $\psi(t)$. The scaling and translation are performed by two parameters: the scale parameter a stretches (or compresses) the mother wavelet to the required resolution, while the translation parameter $b$ shifts the basis function to the desired location. The CWT $C_{s}(a, b)$, of a function $s(t)$ is given as follows (Grossmann and Morlet, 1984):

$$
C_{s}(a, b)=\int_{-\infty}^{+\infty} s(t) \overline{\psi_{a b}}(t) d t,
$$

where the parameters $a$ and $b$ are real, $a>0$ and $\bar{\psi}$ represents the complex conjugate of $\psi$. We define:

$$
\psi_{a b}=\frac{1}{a^{\frac{1}{2}}} \psi\left(\frac{t-b}{a}\right) .
$$

The function $\psi_{a b}$ is obtained from an analysing wavelet $\psi(t)$ by successively applying a change of a time-scale and a change of time-shift. The analysing wavelet should be well-localised in time-frequency and oscillating, so that:

$$
\int_{-\infty}^{+\infty} \psi(t) d t=0
$$

a condition needed to guarantee a perfect reconstruction of the signal (Daubechies, 1992; Meyer, 1993; Torrésani, 1995; Mallat, 2000). Equation (6) is often completed by the more restrictive condition:

$$
\int_{-\infty}^{+\infty} t^{n} \psi(t) d t=0 \quad \text { for } \quad 0 \leq n<p
$$

which states that $\psi$ is orthogonal to polynomials $p_{n}(t)$ of degree $n<p$ (i.e., to have $n$ vanishing moments). This property is needed to detect a singularity eventually masked by regular behaviour represented by a polynomial of low degree.

The wavelet transform is a particularly well-suited tool for studying scaling processes. One can verify that the CWT of scaling processes with stationary increments shares some statistical properties. Indeed, the scale invariance can be reflected by CWT as long as the analyzing wavelet decreases quickly enough to zero (Holschneider, 1995):

$$
C_{s}\left(\lambda a, t_{0}+\lambda b\right) \approx \lambda^{h\left(t_{0}\right)+\frac{1}{2}} C_{s}\left(a, t_{0}+b\right),
$$

where $h\left(t_{0}\right)$ determines the Hölder exponent. Note that the Hölder exponent $h\left(t_{0}\right)$ at any time $t_{0}$ of almost all the realisations of $B_{H}$ is $H$. This important relation establishes the statistical scale invariance of the continuous wavelet transform of self-affine time series, and it constitutes the main property of the CWT used in this study (for more details see Holschneider (1995)). The wavelet coefficients have a short range correlation. Moreover, the greater is the number $p$ of vanishing moments of $\psi$ in Eq. (7) is, the shorter are the correlations. Therefore, the number of vanishing moments is directly linked to the regularity of the wavelet transform. More precisely, Holschneider (1995) has shown that a high regularity of the signal is translated via the CWT from a power-law variation:

$$
\left|C_{s}(a, b)\right| \approx a^{h\left(t_{0}\right)+\frac{1}{2}} \quad \text { when } \quad a \rightarrow 0^{+}
$$

This result confirms that if the stochastic process $s(t)$ is selfaffine or, if it has some long-range dependence properties characterised by the local exponent $h(t)$, then the wavelet coefficients, at a fixed scale, form a stationary process.

In this study, the analysing wavelet $\psi$ is the so-called Morlet wavelet (Grossmann and Morlet, 1984):

$$
\psi(t)=\frac{1}{\sqrt{\pi}} e^{-t^{2}} e^{-2 i \sqrt{\alpha} t},
$$

where $\alpha$ is the shape parameter of the wavelet. The algorithm used for the CWT computation is based on the Technique of Multiple Filtering (TMF, Zaourar et al., 2006), which has a good precision and short computation time, compared with a direct convolution with the analysing wavelet. In the following, we consider $b=t$ the translation applied to each $t$ time point, and the frequency linked to the scale parameter $a, f=2 \sqrt{\alpha} / a$; thus $C_{s}(f, t) \approx C_{s}(a, b)$. The most recent justification of this change of variables is given by Holschneider et al. (2005). Using this change of variables, the CWT is performed in the time-frequency domain and not in the time-scale one. Nevertheless, in the following, we decide to use the term "scalogram" to identify the obtained time-frequency map. The breakdown of the $s(t)$ fluctuations with TMF for all the $f_{n}$ centre frequency uniformly distributed over a logarithmic scale yields $f_{n}=f_{0} 10^{n d}$, where $f_{0}$ is the first central frequency and $d$ is a constant. According to Eq. (8), the scalogram, or the wavelet power spectrum, can be expressed as:

$$
P(f, t)=\left|C_{s}(f, t)\right|^{2},
$$

which, for a sufficiently large frequency, can be re-written as $P(f, t) \approx f^{-\beta(t)}$, where

$$
\beta(t)=2 h(t)+1
$$

The local spectral or scaling exponent $\beta(t)$, estimated by a least-squares fitting of a straight line to each power spectrum plotted in a log-log diagram, describes the local changes of the power law. It can be seen as a measurement of the strength of the singular behaviour of the signal $s(t)$ around a given point $t$. Moreover, the scaling parameter $\beta(t)$ determines the amplitude of the short- and long-term correlations.

\section{Data}

The raw datasets are obtained from three observatories: Chambon-La-Forêt (France), Hermanus (South Africa) and Thule (Denmark). The first two observatories are representative of the mid-latitude geomagnetic field variations, and the third is chosen for a comparison with a 
Table 1. Geomagnetic observatories used in the analysis (in alphabetical order).

\begin{tabular}{|c|c|c|c|}
\hline \multirow{2}{*}{ Name } & \multirow{2}{*}{ IAGA Code } & \multicolumn{2}{|c|}{ Geomagnetic coordinates } \\
\cline { 3 - 4 } & & Latitude N $\left(^{\circ}\right)$ & Longitude E $\left(^{\circ}\right)$ \\
\hline Chambon-La-Forêt & CLF & 49.84 & 85.69 \\
\hline Hermanus & HER & -33.98 & 84.03 \\
\hline Thule & THL & 87.68 & 13.76 \\
\hline
\end{tabular}

Table 2. Main events and their characteristics.

\begin{tabular}{|c|c|c|c|}
\hline Event & MS onset & Min SYM-H/Dst (nT) & Date(s) \\
\hline 15 May 1997 & $05 / 15 / 1997-01: 59$ UT & $-129 /-115$ & $05 / 15 / 1997$ \\
\hline 14 July 2000 & $07 / 14 / 2000-15: 32$ UT & $-347 /-301$ & $07 / 16 / 2000$ \\
\hline March-April 2001 & $03 / 19 / 2001-11: 14$ UT & $/-149$ & $03 / 20 / 2001$ \\
& $03 / 31 / 2001-00: 52$ UT & $/-284$ & $03 / 31 / 2001$ \\
& $04 / 11 / 2001-13: 43$ UT & $/-271$ & $04 / 11 / 2001$ \\
& & $/-114$ & $04 / 18 / 2001$ \\
\hline 6 November 2001 & $11 / 6 / 2001-01: 52$ UT & $/-292$ & $11 / 06 / 2001$ \\
\hline 3-7 September 2002 & $11 / 24 / 2001-05: 56$ UT & & $09 / 08 / 2002$ \\
\hline 28-29 October 2003 & $09 / 07 / 2002-16: 36$ UT & $-168 /-181$ & $10 / 29 / 2003$ \\
& $10 / 28 / 2003-02: 06$ UT & $-103 /-151$ & $10 / 30 / 2003$ \\
& $10 / 29 / 2003-06: 11$ UT & $-390 /-353$ & $10 / 30 / 2003$ \\
\hline 20 November 2003 & $11 / 20 / 2003-08: 03$ UT & $-432 /-383$ & $11 / 20 / 2003$ \\
\hline 7-9 November 2004 & $11 / 07 / 2004-02: 57$ UT & $-394 /-373$ & $11 / 07 / 2003$ \\
& $11 / 07 / 2004-10: 52$ UT & $-271 /-223$ & $11 / 08 / 2003$ \\
& $11 / 072004-18: 27$ UT & $-282 /-289$ & $11 / 09 / 2003$ \\
\hline & $11 / 09 / 2004-09: 30$ UT & & \\
\hline
\end{tabular}

higher latitude station. Additionally, data from the Hermanus observatory are used together with data from 3 other mid-latitude observatories for the calculation of the Dst index. The geomagnetic coordinates of these observatories computed using the IGRF 2005 are given in Table 1. These datasets have been either downloaded from the World Data Center for Geomagnetism in $\mathrm{Ky}-$ oto (swdcwww.kugi.kyoto-u.ac.jp) or the INTERMAGNET website (www.intermagnet.org/apps/dl_data_def_f.php).

We focus on the horizontal component $\mathbf{H}$ of the geomagnetic field, as this is mainly affected by the magnetospheric dynamics.

The main feature of geomagnetic storms is characterised by the Dst index, regularly derived from hourly horizontal magnetic variations recorded at four mid-latitude geomagnetic observatories. In terms of a time series, a geomagnetic storm can be characterized by three phases: initial phase, main phase, and recovery phase. The main phase is characterized by a large decrease of Dst (Gonzalez et al., 1994). A value of Dst $<-50 \mathrm{nT}$ is considered as the threshold criteria to locate the beginning of a magnetic storm.

First, we are interested in the time series of the geomagnetic field from 1 January, 1996, to 31 May, 2005, corresponding to solar cycle 23; more precisely, the hourly mean values provided by the three observatories. In order to better illustrate the monthly perturbations, we analyse separately the increasing phase of solar cycle 23 , starting with 23 October, 1996, and the decreasing phase, starting with 1 July, 2003, and the year 2001, for each of the three observatories. However, here we show the results obtained for the CLF observatory, only. Second, let us note that ten large geomagnetic storms (Dst $<-100 \mathrm{nT}$ ) occurred near the solar maximum period of solar cycle 23. From these events, we select six geomagnetic storms which are investigated in more detail: 15 May, 1997, 14 July, 2000, 3-7 September, 2002, 28-29 October, 2003, 20 November, 2003 and 7-9 November, 2004. The main characteristics of these events are summarized in Table 2. The selected time intervals include the geomagnetic disturbance and a previous period of relatively-low geomagnetic activity. In order to develop this analysis, one-minute sampling data provided at the three magnetic observatories are used. This sampling rate is necessary for a detailed analysis. Quiet and disturbed days are separately considered in the analysis.

\section{Results and Discussion}

\subsection{Scaling behaviour}

The results of the Fourier analysis of the horizontal magnetic variations for increasing phase, decreasing phase, year 2001, July 2000 and October/November 2003, are presented in Fig. 1. For all cases, Fig. 1 shows that the power spectra follow a power-law form typical of a scaling process, with the global exponent $\beta$ corresponding to the slope of the best fitting line in the log-log plot. The spectra displayed in Fig. 1 are continuous and broadband revealing the non-periodic component of the signal. In spite of the variation in the geomagnetic time series from time scales of years to months, all the Fourier power spectra follow a powerlaw behaviour $f^{-\beta}$. It is interesting to observe that there are good scaling regions in all cases indicating that the geomagnetic disturbances are really self-affine fractals. Indeed, the spectral exponent restricted to $1<\beta<3$ is typical 


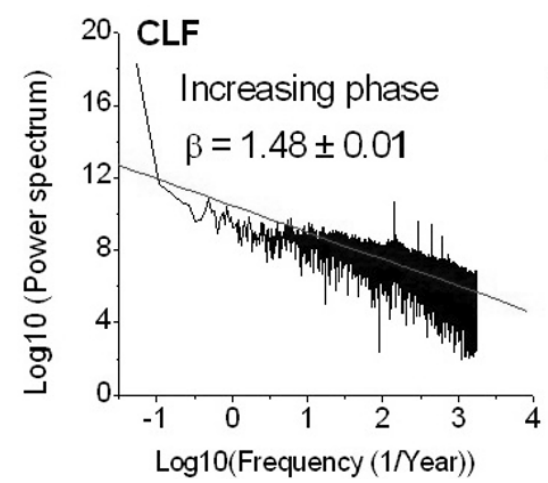

July 2000
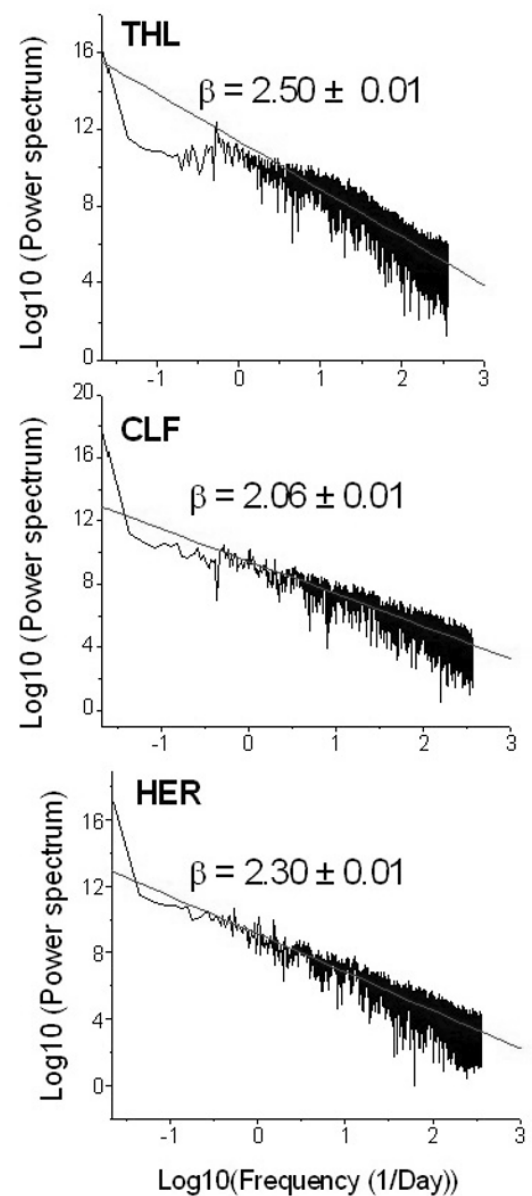

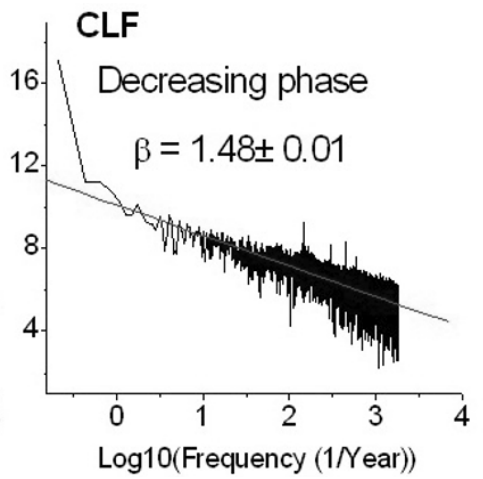

October 2003
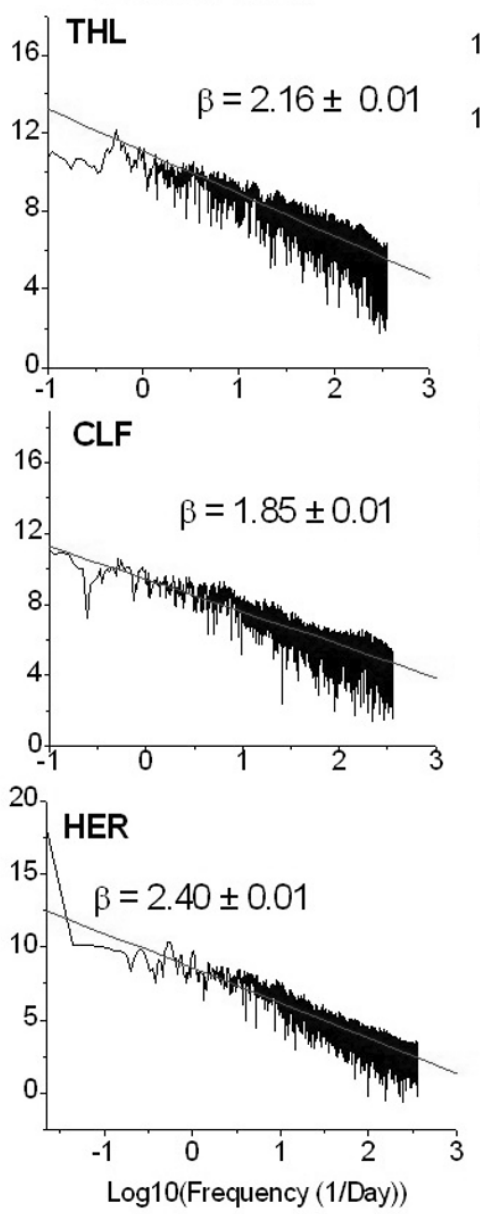

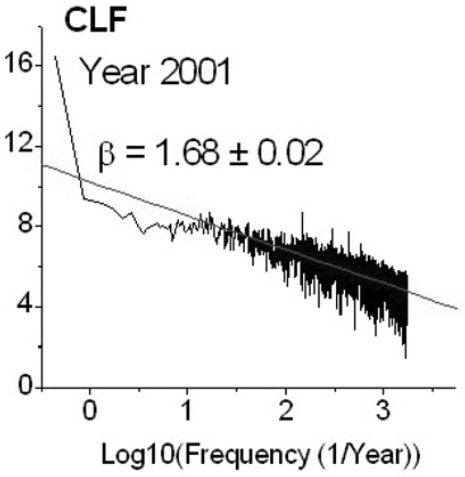

November 2003
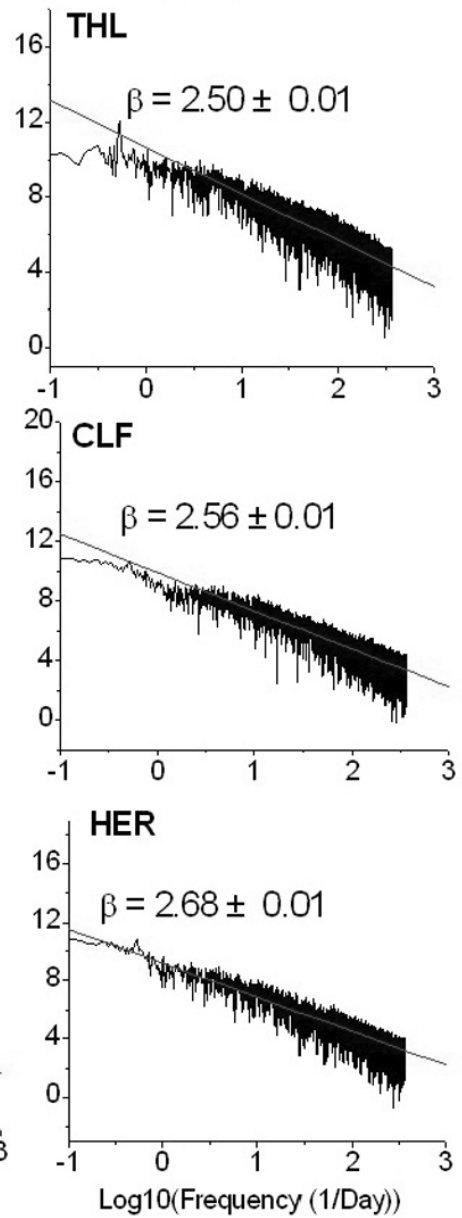

Fig. 1. Fourier power spectra of the geomagnetic variations displaying a scaling behaviour. Top: increasing phase (left), decreasing phase (middle) and year 2001 (right). Bottom: July 2000 (left). October 2003 (middle) and November 2003 (right) obtained at the three observatories. The straight lines are the linear least-squares fit. The slope of the regression line, indicated on each plot, reveals the global exponent $\beta$.

of fBms. As previously discussed, a self-affine time series with a non-zero $\beta$ has long-range (as well as short-range) persistence and antipersistence. The power index seems to be correlated with the geomagnetic activity of the sampled area.

\subsection{Wavelet-based multiscale analysis of magnetic} storms

We have applied the wavelet-based approach to analyze the geomagnetic time series observed in a sequence from time scales of years to months using the sampling intervals described above. Wavelet analyses have no inherent problems such as those in Fourier power-spectral analysis, such as windowing, detrending, etc. As we have already pointed out, by considering analysing wavelets that make the wavelet transform blind to the low-frequency trends, any bias in the raw data can be removed and the existence of power-law correlation with scale-invariance properties can be revealed accurately. The stochastic component of a time series has two main aspects, its statistical distribution of values and its persistence. The persistence, characterized by $\beta$, measures the correlation between adjacent values within the time series: it can be strong $(\beta>1)$ or weak $(\beta<1)$ (Malamud and Turcotte, 1999). Values of a time series can affect other values in the time series that are not only close in time 

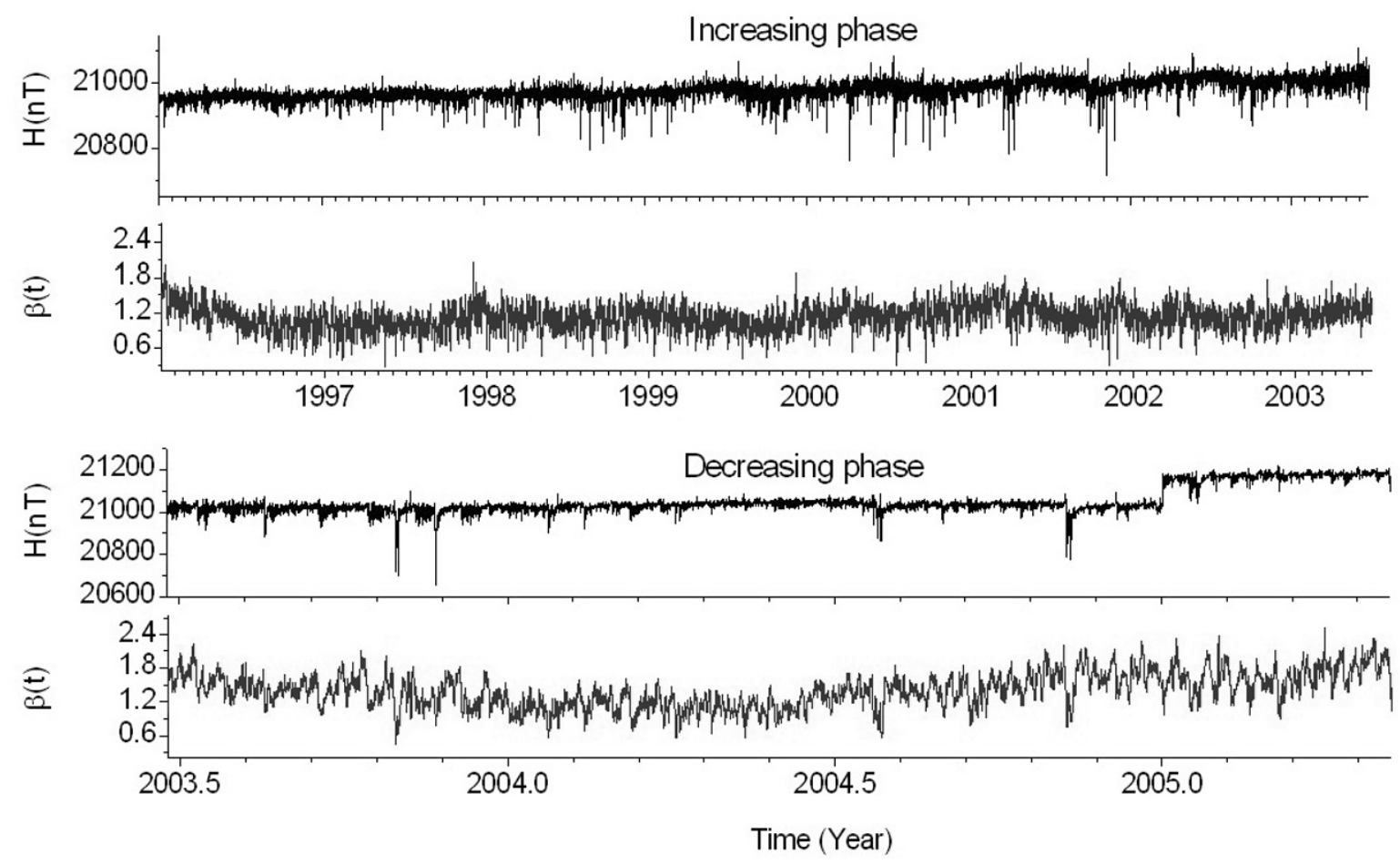

Fig. 2. Horizontal component temporal behaviour, as seen by the hourly means, during increasing phase (top) and decreasing phase (bottom) of the solar cycle 23 at the CLF observatory. The corresponding dynamics of wavelet spectral exponents are also plotted.

(short range), but also far away in time (long range). Since self-affine time series have a power-law dependence of the power-spectral density function on frequency, they exhibit long-range persistence.

4.2.1 Chambon-La-Forêt: a test dataset During the first step of this analysis we use the 81920 values recorded in the CLF observatory and corresponding to both phases of solar cycle 23. As Fig. 2 shows, the geomagnetic time series display an heterogeneous scaling behaviour exhibiting sharp singularities and is, therefore, suitable for a multiscale analysis based on a power-law exponent estimation. We also refer to this figure the results of the processing by CWT of both phases. It can be clearly seen that the temporal variations of the spectral exponents $\beta(t)$ indicate up more irregularities, characterized by strong fluctuations compared to the flatness of their measurements.

To show better the variations of the six chosen storms, we plot (Fig. 3) several portions representing one-year variations at the CLF, except close to the solar cycle decreasing phase, i.e. July 2003, when we use data over a half year, only. Indeed, the scaling exponents delineate clearly both the strong disturbances corresponding to the magnetic storms. In all these cases, we observe that correlations between the geomagnetic time series and their spectral exponents show that the lowest values of $\beta(t)$ coincide with the most perturbed moment. The estimated average exponent values $\beta_{a} \approx 1.11 \pm 0.20$ for the increasing phase, and $\beta_{a} \approx 1.40 \pm 0.31$ for the decreasing phase, indicate globally an antipersistent behaviour in the time series.

\subsubsection{Extended analysis \\ 2001 Magnetic Storms: One-year window}

For a detailed analysis using hourly-mean values, we have examined the geomagnetic time series recorded at the CLF, HER, and THL observatories during the year 2001, characterized by two important events. Our goal is now to make a comparative study of the wavelet spectral exponent deduced from the geomagnetic time series with the Dst index scaling behaviour studied by Balasis et al. (2006). We would like to note that this study deals with the analysis of raw time series recorded in geomagnetic observatories. In this context, the used data have not been preprocessed (e.g. filtered) prior to the wavelet analysis. Therefore, the scaling exponent values may fluctuate from the values specifically known for the quiet periods (i.e., when $1<\beta(t)<2$ is expected) or for the disturbed periods (i.e., when $2<\beta(t)<3$ is expected). This can clearly be seen in Fig. 4, where the $\beta(t)$ values for the Dst index time series are also represented. The $\beta(t)$ values corresponding to Dst variations display a more robust, or disciplined, structure (i.e., they fall better within the theoretically predicted intervals $(1,2)$ for antipersistency and $(2$, 3 ) for persistency). Two classes of signal have been widely used to model stochastic fractal time series (Heneghan and McDarby, 2000): fractional Gaussian noise (fGn) and fractional Brownian motion (fBm). These are, respectively, generalizations of white Gaussian noise and Brownian motion. A formal mathematical definition of continuous $\mathrm{fBm}$ was first offered by Mandelbrot and Ness (1968). For the case of the fGn model, the scaling exponent $\beta$ lies between -1 and 1 , while the regime of $\mathrm{fBm}$ is indicated by $\beta$ values from 1 to 3 .

The scaling exponent $\beta$ is related to the Hurst exponent, $H: \beta=2 H+1$ with $0<H<1(1<\beta<3)$ for the fBm random field model (Heneghan and McDarby, 2000). 

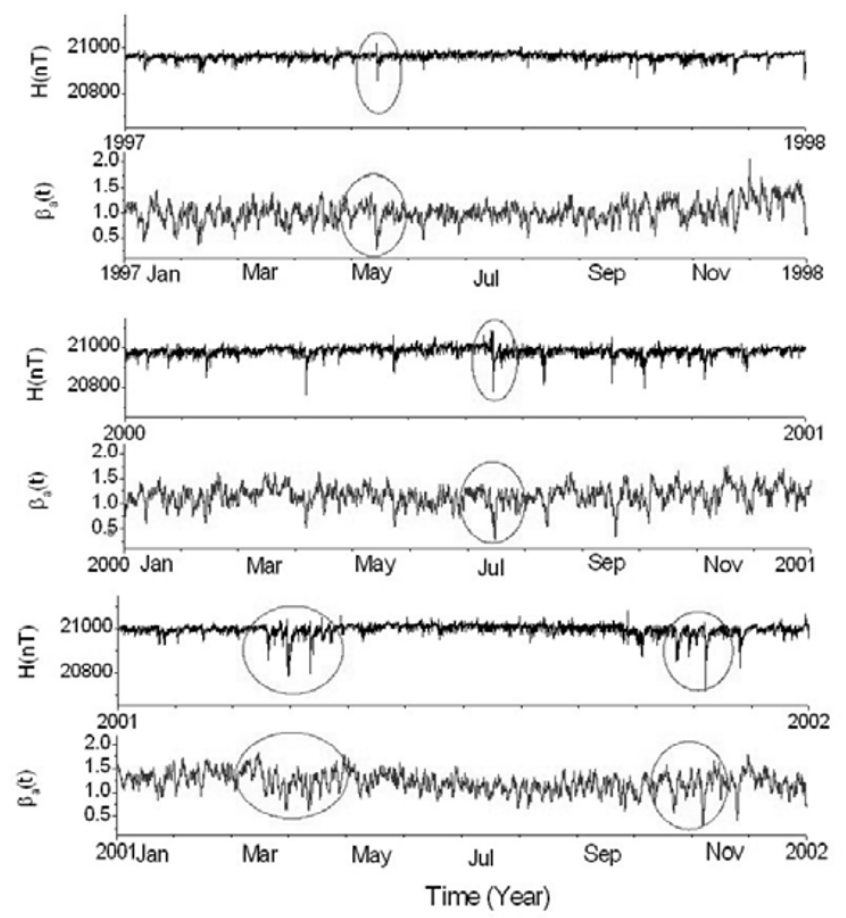
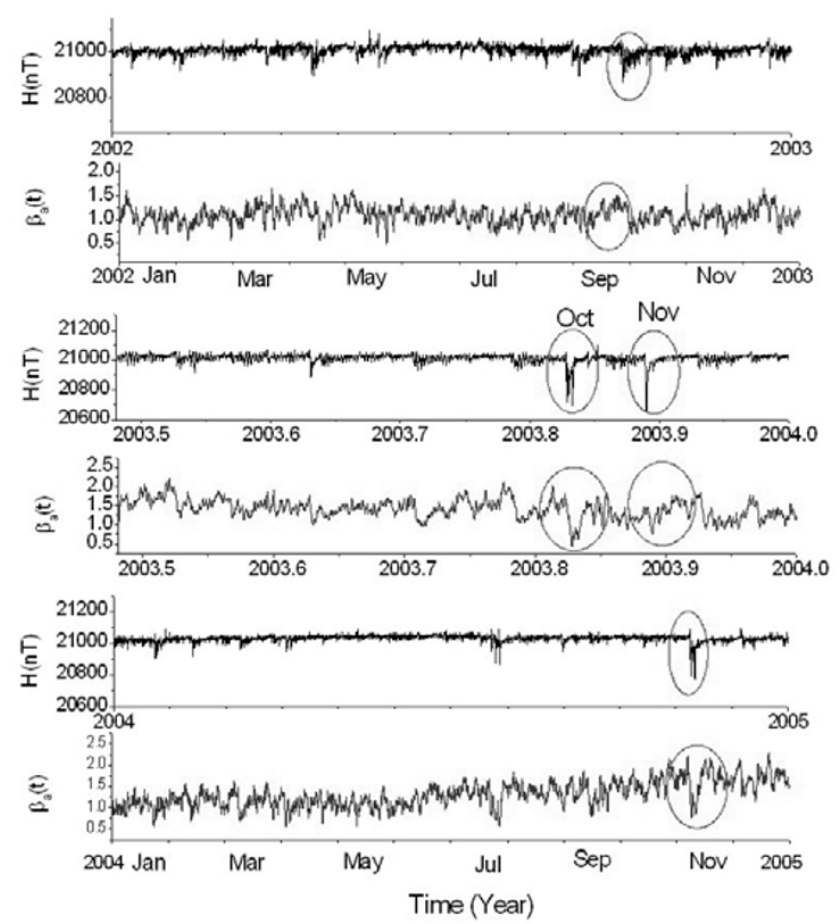

Fig. 3. Horizontal component temporal behaviour, over one year, at the CLF observatory. The corresponding dynamics of wavelet spectral exponents are also indicated. Geomagnetic storms considered in this study are underlined by ellipses.
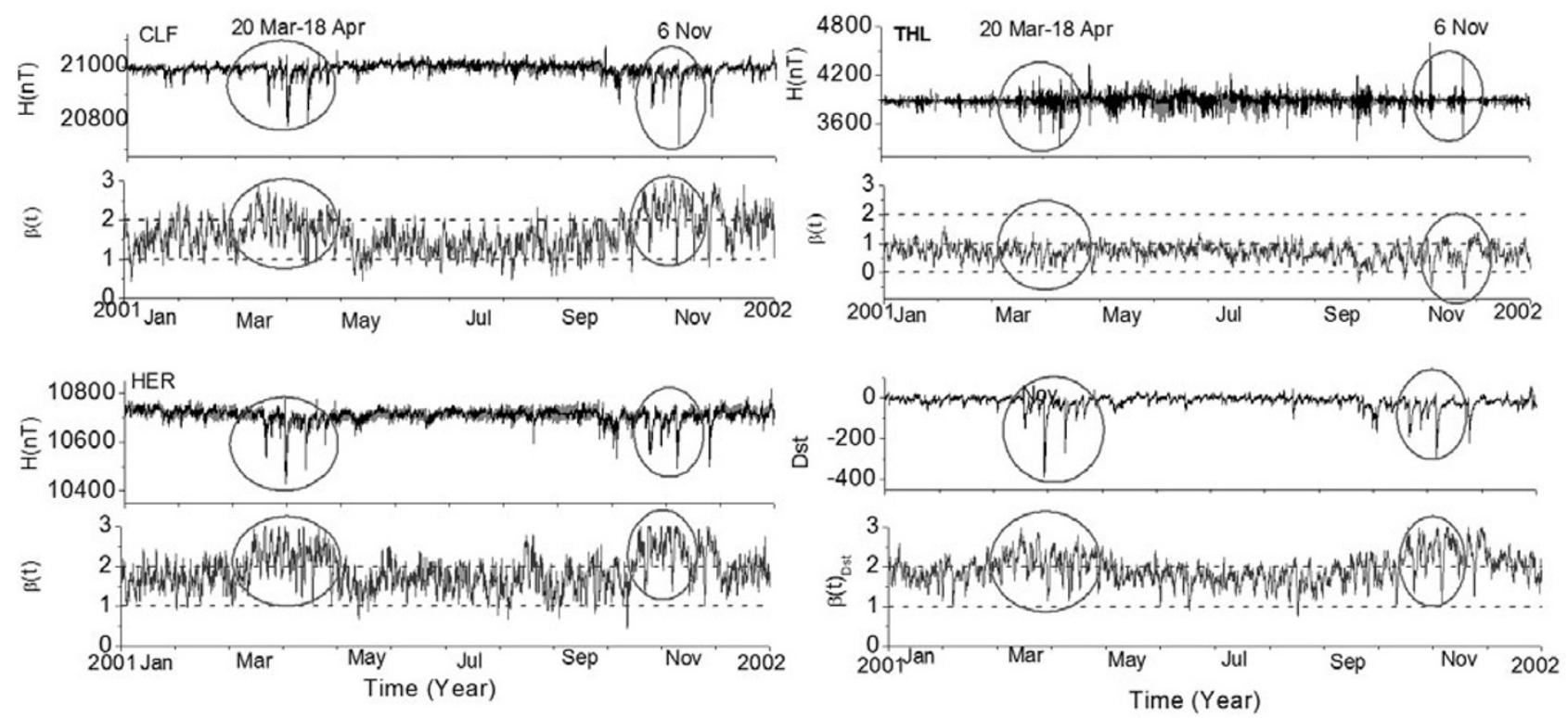

Fig. 4. Horizontal component temporal behaviour, as seen by hourly means, at CLF, HER, and THL, and their corresponding dynamics of wavelet spectral exponents. Dst index and its spectral exponents are also plotted. Ellipses show storms noted in March, April and November 2001.

The exponent $H$ characterizes the persistent/antipersistent properties of the signal (Balasis et al., 2006, 2008, 2009, 2011). The range $0<H<0.5(1<\beta<2)$ indicates antipersistency, which means that if the fluctuations increase in a period, it is likely to decrease in the interval immediately following and vice versa. Physically, this implies that fluctuations tend to induce stability within the system (negative feedback mechanism). When the time series exhibits persistent properties, i.e., $0.5<H<1(2<\beta<3)$, it means that if the amplitude of the fluctuations increases in a time interval, it is likely to continue increasing in the next immediate interval. In other words, the underlying dynamics is governed by a positive feedback mechanism. The value $H=0.5(\beta=2)$ suggests that there is no correlation between the repeated increments. Consequently, this particular value takes on a special physical meaning: it marks the 
transition between antipersistent and persistent behaviour in the time series.

Let us mention that the Dst index is calculated as an average of four mid-latitude geomagnetic observatories after taking into account the secular variation and the system of the external $\mathrm{Sq}$ currents at each location. Consequently, the Dst index catches corrections applied to the magnetic field measurements with respect to these influences [http://wdc.kugi.kyotou.ac.jp/dstdir/dst2/onDstindex.html], whereas the observatory data, to which the wavelet transform has been applied, are raw measurements.

In Fig. 4, one can observe that all fluctuations have a signature in terms of the scaling spectral exponent $\beta(t)$ computed by linear regression fitting of the scaling region. Mainly, the intense geomagnetic storms recorded (between 20 March-18 April, and 6 November, 2001), are well identified by the spectral exponents. $\beta(t)$ curves are characterized by a slightly different behaviour and a high smoothness level, comparable with the previous ones (see Fig. 3). Indeed, Fig. 4 shows that the $\beta(t)$ values are mostly distributed in the range $[0.6,3]$ probably indicating the presence of fractional Gaussian noise (fGn) for $0<\beta(t)<1$, and $\mathrm{fBm}$ for $1<\beta(t)<3$. However, for the CLF and HER observatories, $\beta(t)$ emphasize persistent properties $2<\beta(t)<3$ around both 20 March and 18 April, and $\beta(t) \approx 3$ in the region of 6 November, 2001. The smaller values $(\beta(t)<2)$ coincide with peaks probably correlated to the start of the storm main phase, indicating antipersistence properties. However, as outlined by Richardson and Zhang (2008), intense storms (Dst $<-100 \mathrm{nT}$ ), such as the ones studied in this work, may be more complicated. The large deviation of the scaling parameter values (Fig. 4) are indicated at the transition from antipersistent to persistent behaviour, signifying an episode of a disturbed period. As mentioned above, this means that if the amplitude of the fluctuations increases in a time interval, it is likely to continue increasing in the next immediate interval. Thus, the underlying dynamics is governed by a positive/negative feedback mechanism. Figure 4 also shows the hourly Dst values revealing the geomagnetic storm and associated spectral wavelet exponent, outlining the transitions from antipersistent to persistent behaviour related to storms around 20 March-18 April, and 6 November, 2001. These observations are close to those in Balasis et al. (2006) showing that a spectral signature associated with the emergence of persistency of the Dst time series precedes an intense storm (here, the storms on 31 March, 2001 and 6 November, 2001, with a minimum Dst of $-387 \mathrm{nT}$ and $-292 \mathrm{nT}$, respectively).

To better assess the potential of the local spectral exponent $\beta(t)$ to delineate and characterise transitions in magnetograms, we also examine the time series accompanying the intense geomagnetic perturbations that occurred on 2930 October, 2003, and 20 November, 2003. We develop this analysis, using one-minute time series obtained at the CLF, HER and THL observatories. In this case, the smallest resolvable frequency used is $f_{\text {min }}=0.0223 \mathrm{day}^{-1}$, the largest is $f_{\max }=724$ day $^{-1}$ and the number of samples is 87840 .

2003 Magnetic Storms: 2-month window
Figure 5 shows the scalograms obtained by using continuous wavelet analysis at CLF and HER (middle latitudes) during 29-30 October, and 20 November, 2003. These results are also compared with THL (high latitude) data. These three scalograms exhibit the energy distribution of the wavelet coefficients in the time-frequency plane. As stated previously, the wavelet transform consists of calculating a 'resemblance index' between the signal and the wavelet. If a signal is similar to itself at different scales, then the 'resemblance index', or wavelet coefficients, will be similar at different scales. We note that disturbance periods are characterized by higher wavelet coefficients as is the case for both intense geomagnetic storms of 29-30 October, and 20 November, 2003. When a geomagnetic storm is under development, the horizontal component of the geomagnetic field recorded in the magnetograms (see Fig. 6) can be represented by very roughness functions, and accordingly the energy of the wavelet coefficients is significantly large. On the other hand, when the magnetosphere is under quiet conditions, the wavelet coefficients exhibit very small energy. One can observe that the magnetic observatory data do not show the same singularity pattern. This behaviour may be related to differences in the magnetic coordinates, local time, ground conductivity and current effects. The scalogram can enable the quiescent and non-quiescent periods in the horizontal component to be identified independently of the general signature of the analyzed series. Figure 5 underlines that for a higher-latitude magnetic observatory (THL), larger energy wavelet coefficients are more frequent at a high frequency (enhanced by the red color). This is probably due to the presence of strong ionospheric currents at high latitudes. For mid-latitude stations (CLF and HER), the behaviour of the energy coefficients is dominated by low energy at a low frequency. This indicates an attenuation of the high-frequency content in the analysed signal provided by mid-latitude observatories.

We have also investigated the temporal evolution of spectral exponents (Fig. 6). This figure underlines the extraordinary complexity of the geomagnetic time series variations. For all observatories, the spectral exponents clearly corroborate the fractal pattern of the analysed time series and their different scaling characteristics. Besides strict locality, the estimated spectral exponents have the advantage of providing remarkable maps of singularity structures in data. Various transitions induced by the intense geomagnetic activity during this period are better differentiated by $\beta(t)$ transitions than the associated magnetograms represented in Fig. 6. Once again, we observe that the amplitude fluctuations vary with the observatory magnetic coordinates, viewing a slight decay in spectral exponent fluctuations towards lower latitudes (HER compared with THL). For all three observatories, the exponent value curves appear smoother from a high to a low latitude, when analyzing data according to their geographical locations, with $\beta(t)$ values in $[1,3]$. These values confirm that the geomagnetic time series are qualitatively analogous to $\mathrm{fBms}$. This is expected to lead to either persistence or antipersistence in the system. Indeed, as can be distinguished in Fig. 6, the dynamics of the exponent time series shows a spiky activity when $\beta(t)$ values increase indicating a transition to an intense storm. In the 


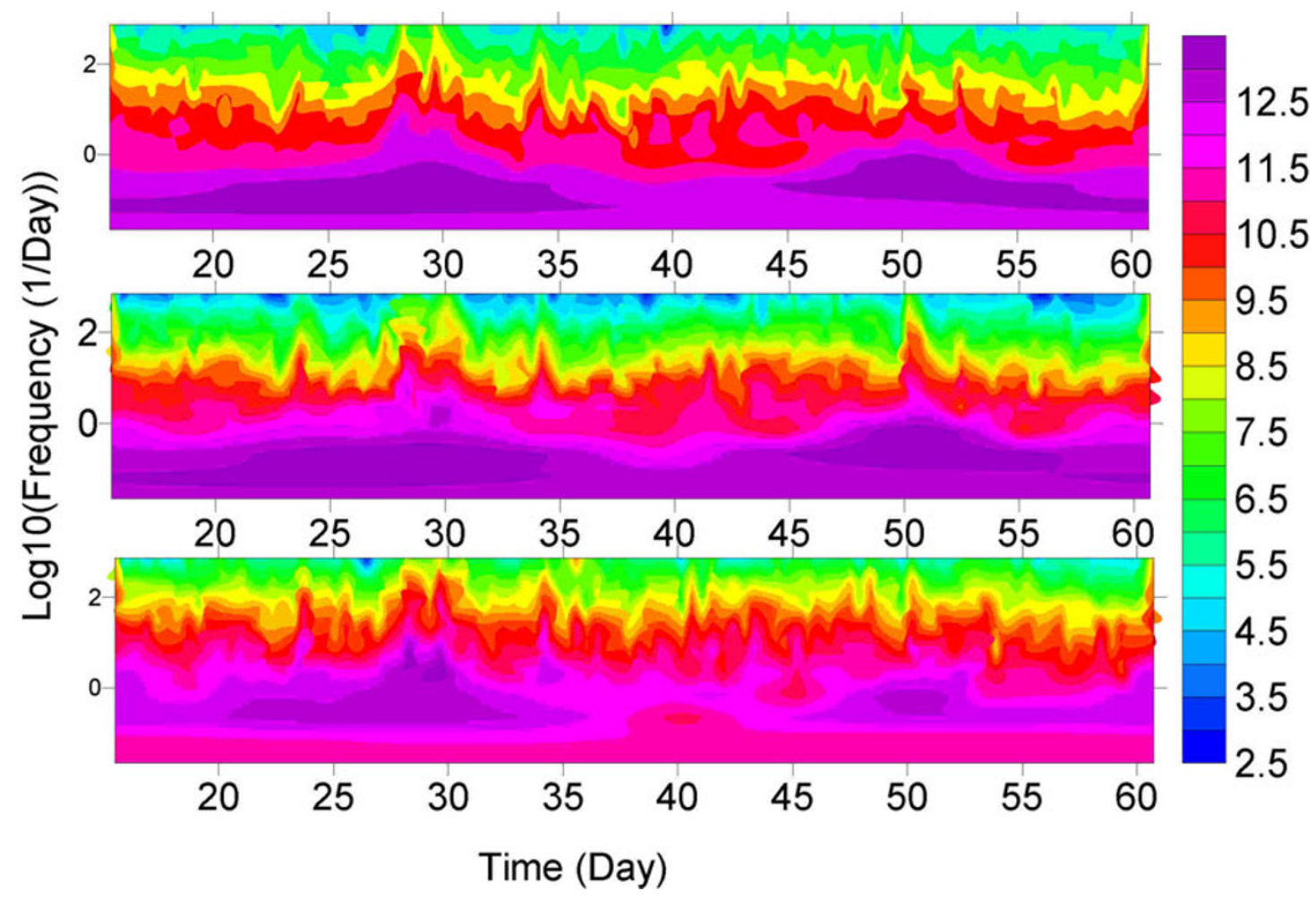

Fig. 5. Three scalograms revealing the energy distribution of the wavelet coefficients of the horizontal component. The time series start on 1 October, 2003, and comprise storms from October and November 2003. The three observatories are CLF (top), HER (middle) and THL (bottom). The color scale reflects the increasing energy from blue to violet.

next interval, when $\beta(t)$ values decrease, the geomagnetic field activity corresponds to a quiet period. As mentioned previously, such a rapid change is typical for a system starting a phase transition or attaining a new shape.

\subsection{An activity estimator based on the spectral expo- nent}

\section{Magnetic Storms: 3-day window}

To better visualise the nature of the transition described by the temporal evolutions of $\beta(t)$ curves, we illustrate in Fig. 7 a zoom covering a period of three days around the storms of 29-30 October, and 20 November, 2003. For this analysis, one-minute values are also used. Indeed, rather than searching for the existence of a correlated behaviour over the complete geomagnetic time series, we identify a "local measurement" of the degree of long-range correlations described by variations of the scaling exponent during an intense geomagnetic storm. We also compare the temporal history of these storms as measured by magnetograms and the SYM-H time series. Let us recall that the SYM$\mathrm{H}$ index, measured in $\mathrm{nT}$, is a ground-based magnetic index developed to describe geomagnetic disturbance fields at middle- and low-latitudes with a high-time resolution (Wanliss, 2005a; Wanliss and Showalter, 2006). It is the oneminute high-resolution version of Dst, derived using data from magnetic observatories close enough to the magnetic equator, so as not to be influenced by auroral current systems. The SYM-H index provides an excellent measure of the large-scale behaviour of the ring current and magnetic storm dynamics (see Iyemori, 1990).

Figure 7 shows the boundaries between antipersistent/persistent behaviour reflected by the scaling exponent $\beta(t)$. For the two observatories, the spectral exponents picture the detailed features contained in this interval, bearing a dynamical transition from quiet times to intense storms.

For the SSC on 29 October, 2003, at 06:12 UT, marked by a vertical dashed line in Fig. 7, the scaling exponent exhibits a rapid change from an antipersistent to persistent value $\sim 2.3$ at the storm onset as indicated by the transition phase T1. During the period 09:00 UT-13:00 UT corresponding to the initial part of the main phase, the exponent decreases abruptly reaching a minimum value of $\beta(t) \approx 1$. The second transition phase T2 of the exponent, beginning at 13:00 UT, is associated with the intense main phase depression restarted with the decrease of the geomagnetic time series from 13:00 UT until 24:00 UT. Indeed, the peak intensity in the disturbance occurred at 24:00 UT to $-350 \mathrm{nT}$ in SYM$\mathrm{H}$ is shown with a gradual increase of the spectral exponents reaching a maximum value $\beta(t) \approx 3$ at the CLF and HER observatories. During the recovery phase, the scaling exponents steadily fluctuate around a mean value $\beta(t)>2$, indicating that the magnetospheric response to the reduced solar wind energy input is statistically similar.

The intense storm on 30 October, 2003, at 16:20 UT marked by a vertical dashed line in Fig. 7 is remarkable in that the exponent exhibits a phase transition T3 at 20:00 UT and reaches a value $\beta(t) \approx 2.5$, consistent with the development of a sharp main phase (SYM-H $\approx-400 \mathrm{nT})$ which commenced at 18:00 UT on 30 October. Another change of scaling exponent from antipersistent to persistent values, indicated as transition T4, is observed at 02:00 UT at the CLF, and 04:00 UT at the HER, observatories, respectively. Referring to this figure, a sudden increase in the $\mathbf{H}$ com- 


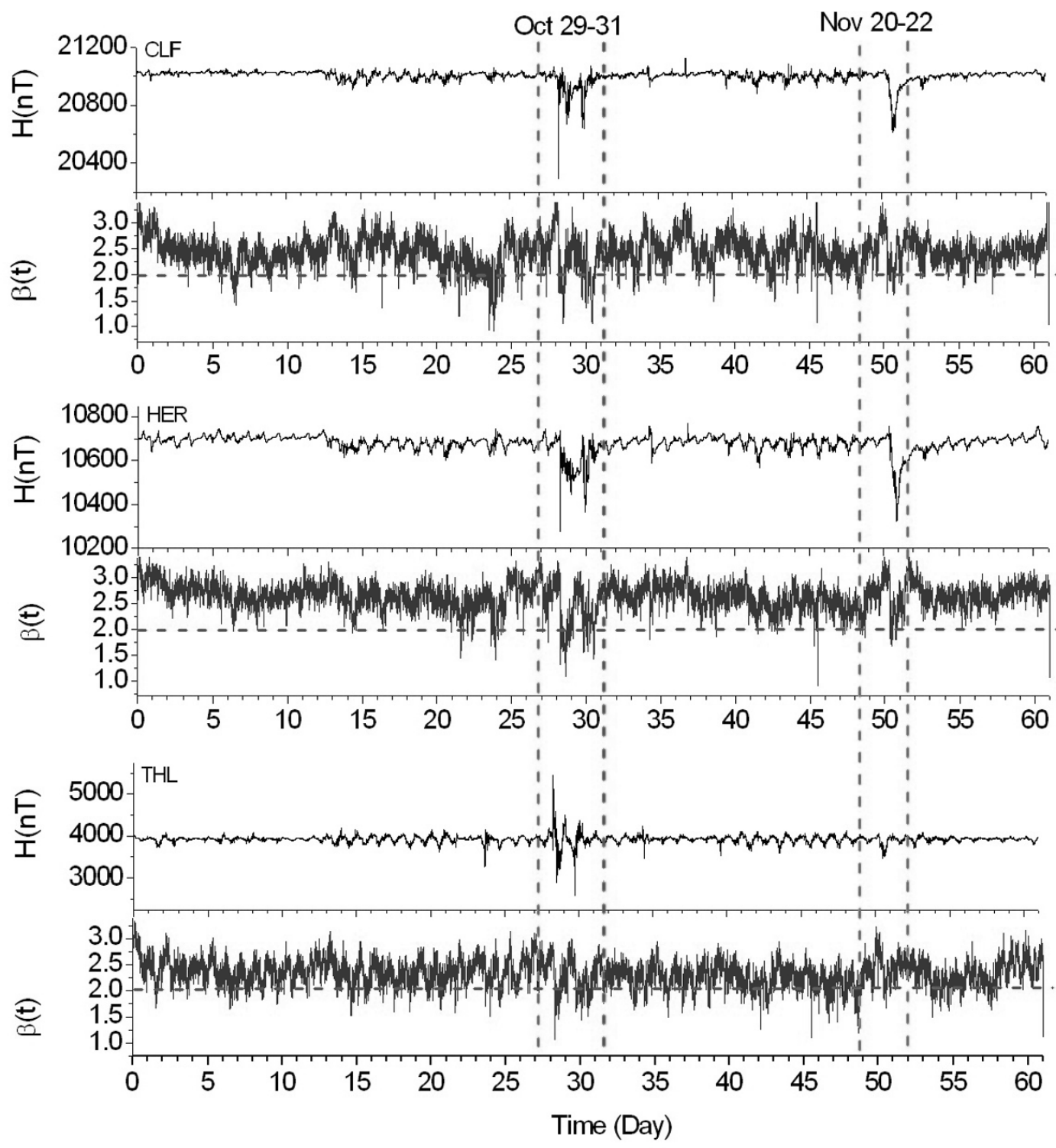

Fig. 6. Horizontal component temporal behaviour, as seen by one-minute values, in the CLF, HER and THL observatories during the October-November 2003 time interval and the associated wavelet spectral exponents. The vertical dashed lines show the intense storms of October and November 2003.

ponent is observed at 31 October, for approximately $6 \mathrm{~h}$ with peaks at 05:00 UT and 11:00 UT at the CLF and HER observatories, and is of noticeable magnitude in the 1-min SYM-H. This enhancement in the magnetic field is due to the occurrence of an interplanetary shock at 04:51 UT. Evidence of the ground magnetic variations of the $\mathbf{H}$ component is clearly marked by the spectral exponents, as shown by the transient phases T5 and T6 from antipersistent to persistent values around the start of the principal phases, except for the transition phase T5 at the CLF observatory, which is characterised by antipersistent behaviour.

It is clear from Fig. 7 that during active intervals, the SYM-H index reaches large negative values. Variations of the two spectral exponents exhibiting a relatively sudden change from a less-correlated to a more-correlated pattern of multiscale fluctuations at storm onset reflect variations in the intensity of the ring current dynamics (Wanliss, 2005b).

All these observations are consistent with the powerful solar flares and CMEs which occurred during October-
November 2003 (Alex et al., 2006). The "Halloween" 2003 magnetic storm (29 October, 2003-31 October, 2003) was a rare event that provided an ideal set of conditions to examine magnetospheric/ionospheric responses to the solar wind (Balasis et al., 2012). The Halloween 2003 storm was a result of an interaction between a strong solar wind due to a solar flare and the Earth's magnetosphere. The solar flare occurred on 28 October, 2003 at 11:00 UT, accompanied by a massive jet of ionized particles (Farrugia et al., 2003; Gopalswamy et al., 2005). Another major solar flare occurred on 29 October that affected the Earth's magnetosphere on 30 October causing magnetic storms, while effects of the previous eruption still continue and are causing unusual aurora borealis. The rapid CME from the X10 flare on 29 October at 20:49 UT generated a shock that impacted the Earth's magnetosphere and gave rise to an enhancement in the horizontal component of the geomagnetic field at 16:20 UT on 30 October, 2003.

The intense event of 20 November, 2003 (corresponding 

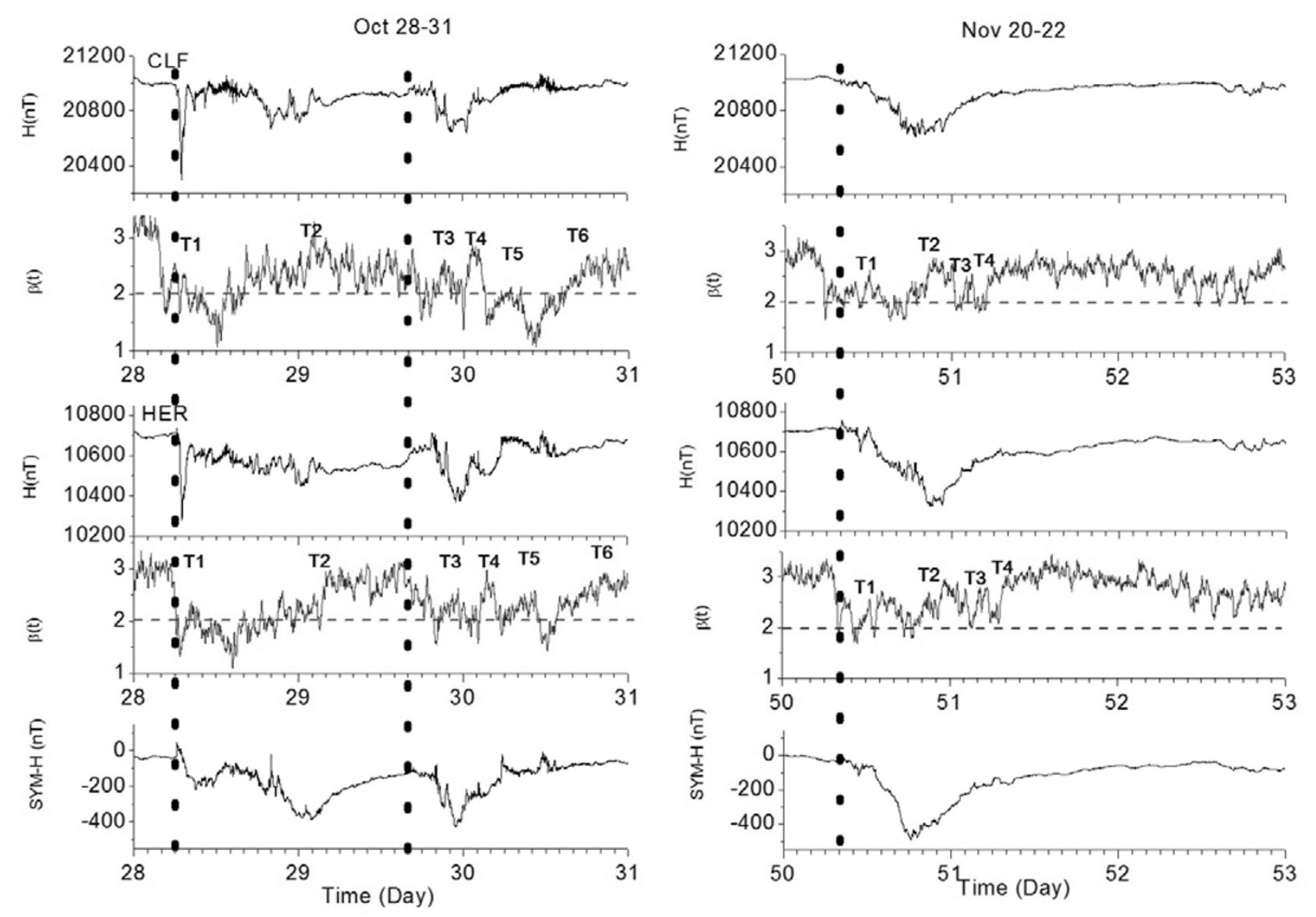

Fig. 7. Horizontal component temporal behaviour, as seen by one-minute values, around the storm of 28-29 October (left), and 20-21November, 2003 (right) at CLF and HER, and the associated wavelet spectral exponents. The SYM-H index is also plotted. The vertical dashed lines delineate the storm onset. Labels T1 to T6 above the scaling exponent indicate the phase transition near the storm onset. Minor ticks on the abscissa equal two-hour intervals. The horizontal dashed lines indicate the transition from an antipersistent $(1<\beta<2)$ to persistent $(2<\beta<3)$ behaviour of the field.

to day number 51) at 08:03 UT, remarkable in Fig. 7 (right) as transitions $\mathrm{T} 1$ and $\mathrm{T} 2$ in the scaling exponent, occurred in the development of an intense main phase of the storm as seen in the $\mathbf{H}$ component at the CLF and HER observatories. During the recovery phase the scaling exponents steadily fluctuate around a mean value $\beta(t)>2$. A gradual decrease of the scaling parameter is observed from 22 November (day 53). It is worth mentioning that with the SYM-H index characterized by a poor temporal localization of events, wavelet analysis reveals all transitions accompanied by specific signatures in the temporal scaling of fluctuations in a storm-time geomagnetic time series. Indeed, transitions T3 at 02:00 UT and T4 at 06:00 UT, respectively, observed in the scaling exponents, feature small changes in the SYM-H.

\section{Four Magnetic Storms: 4-day window}

It is also worthwhile mentioning that the same analysis is reproduced for events as those of 15 May, 1997, 14 July, 2000, September 2002 and 7-9 November, 2004, at the two CLF and HER observatories. A similar behaviour has also been detected, and other singularities have been found, in that signal.

As illustrated in Fig. 8(a), the other four storms feature the same general behaviour; each case features a smaller scaling exponent decreasing toward 2 preceding the storm onset, and a progressive increase to a larger value once the storm has begun. However, this scenario is not exactly described in the same way by the three observatories.

In the case of the storm of 15 May, 1997, occurring around 1:58 UT, as shown in Fig. 8(a), the scaling exponent at the CLF observatory increases, and fluctuates around the value $\beta(t) \approx 2.5$ during the 6 hours corresponding to the initial part of the main phase, which is identified with the SYM-H index. The first peak of the storm, occurring around 8:10 UT, is outlined by a decreasing value of the scaling exponent towards 2 followed by a progressive increase to a value $\beta(t) \approx 2.5$ during the main phase of the storm. For the HER observatory, the phase transition marked $\mathrm{T} 2$ is clearly localized by the scaling exponent, displaying a persistent behaviour reaching a maximum value of $\beta(t) \approx 3$ at 19:00 UT. The gradual fall of $\beta(t)$, from a persistent to an antipersistent regime is observed after 16 May, when the magnetic field at the Earth returns to normal conditions.

The terrestrial ring current dynamics during the time interval of 13-18 July, 2000, consists of two small-tomoderate geomagnetic storms followed by an intense storm. Extreme geomagnetic activity during this period was caused by three interplanetary coronal mass ejections (ICME) each driving interplanetary shocks, the last shock being very 
(a)
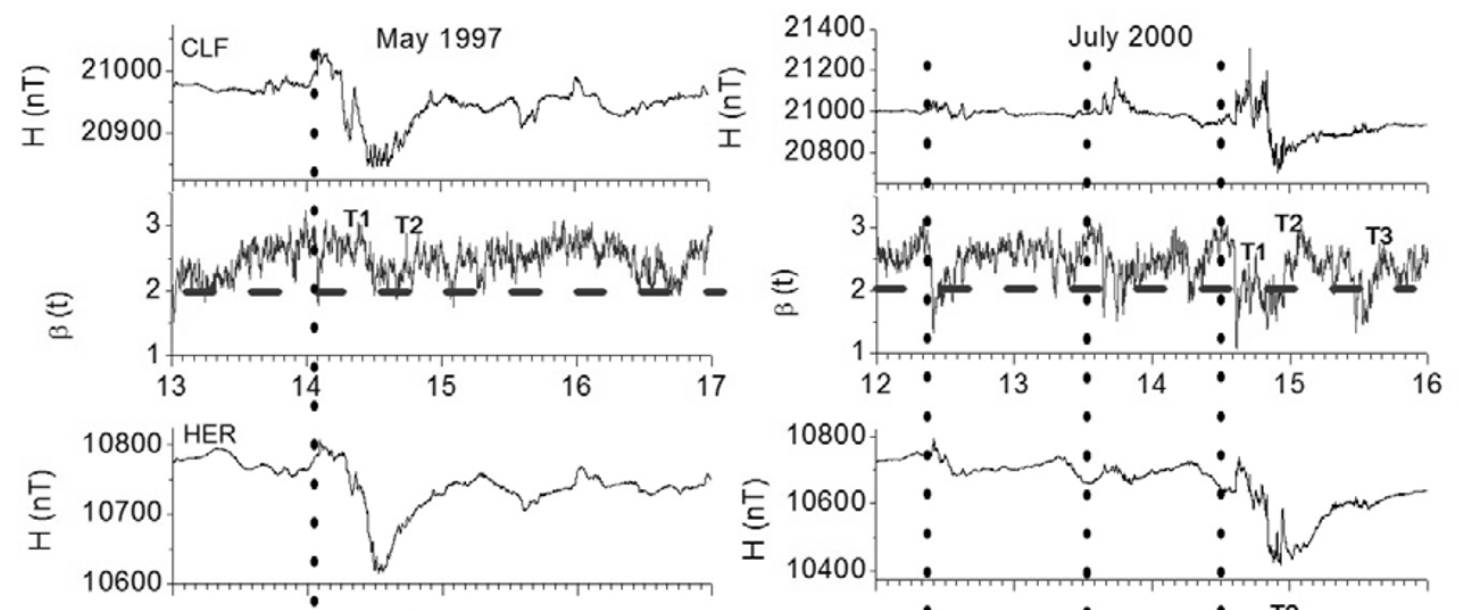

$\underbrace{}_{0}$
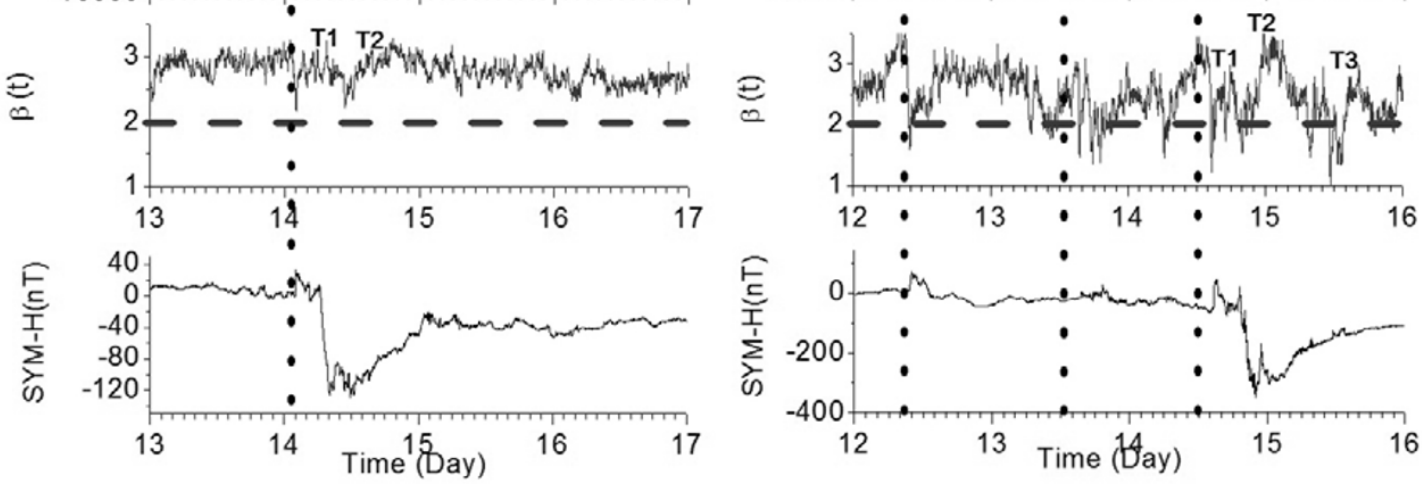

(b)
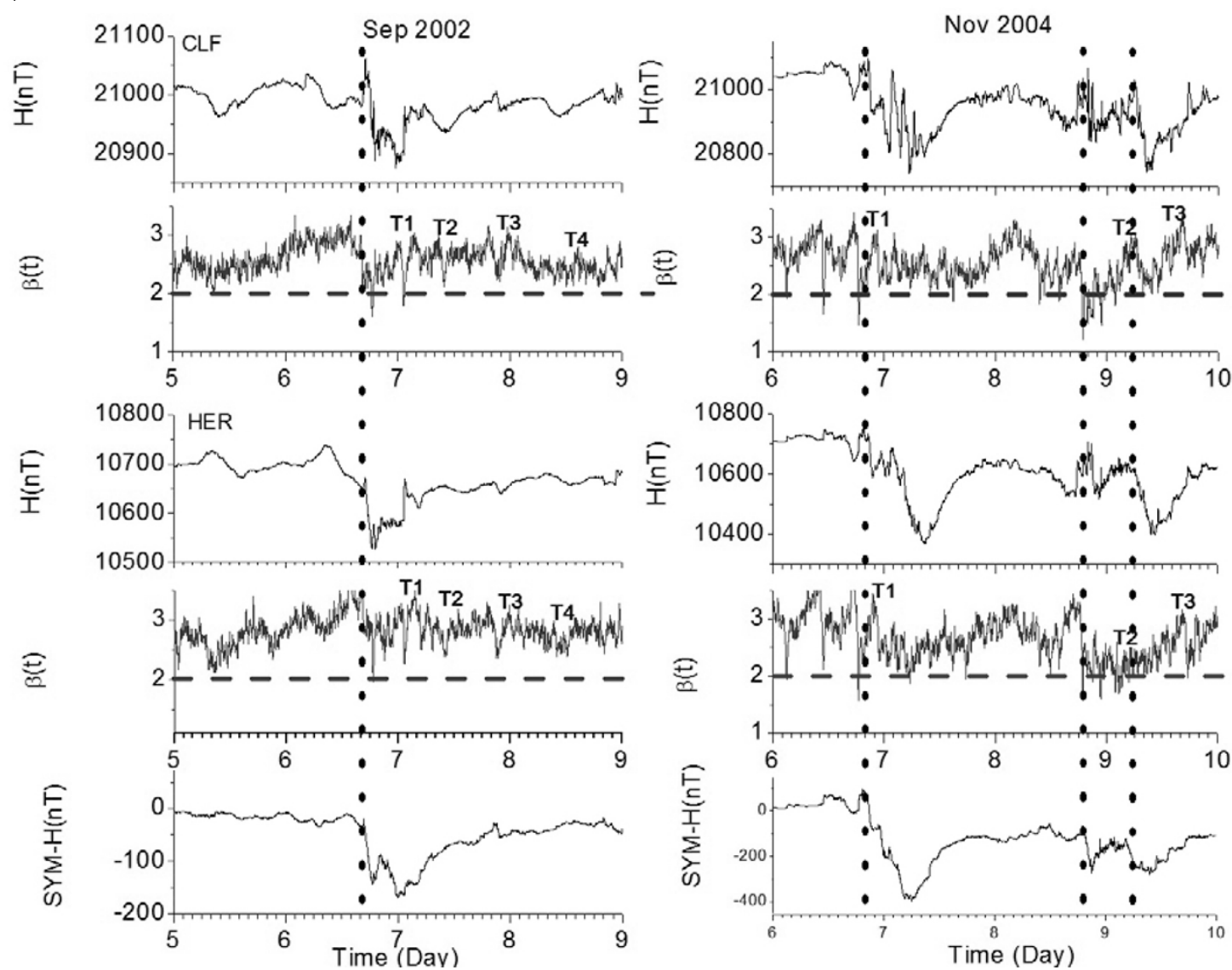

Fig. 8. Horizontal component temporal behaviour, as seen by one-minute values at the CLF and HER observatories, and associated wavelet spectral exponents. (a) Storms of 15 May, 1997 (left) and 15 July, 2000 (right). (b) Storms of 7 September, 2002 (top and left) and 14 November, 2004 (right). 
strong and reaching Earth at 14:40 UT on 15 July, as indicated by the variations in the geomagnetic $\mathbf{H}$ components and the SYM-H time series (Fig. 8(a), right). Dashed lines indicate the onset of the principal geomagnetic events. Note that the different storm phases are identified according to the distribution of the SYM-H index. The associated scaling exponents at the CLF and HER observatories shown in Fig. 8(a) (right) exhibit some transitions from prior to the storm onset to a rapid jump to close to 3. Compared with the SYM-H index, the different storm onsets: 09:00 UT 13 July, 15:00 UT 14 July, 14:00 UT 15 July, and 15:00 UT 16 July, are identified simply according to the distribution of the spectral exponents. In all cases, the storm onsets are indicated by rapid changes from antipersistent to persistent values, defining a dynamical phase transition.

As shown in Fig. 8(a) (right) the strong magnetic storm of 14:00 UT 15 July is clearly described by the scaling exponents as the first transition phase $\mathrm{T} 1$ of the initial main phase onset around 17:00 UT, followed immediately by another transition $\mathrm{T} 2$ reaching a maximum value of $\beta(t) \approx$ 3 and maintains this value until 02:00 UT corresponding to the end of the main phase on May 16. Note that the transition T3 (beginning at 18:00UT) of the exponent from an antipersistent to persistent value, $\beta(t) \approx 3$, corresponds to the decreasing value observed in the magnetograms at the CLF and HER observatories.

For the storm on 7 September, 2002 (Fig. 8(b) left), spectral exponents $\beta(t)$ feature an increasing phase T1 around 18:00 UT and reach a maximum value at 00:00 UT on September 8, coinciding with the period of the main phase of the storms. Interestingly, the three minimum peak values clearly noticed on magnetograms, around 11:00 UT and 22:00 UT on September 8, and around 15:00 UT on September 9 , are indicated by an abrupt increase of $\beta(t)$, marked T2-T4 on Fig. 8(b) left.

The intense disturbed period during 7-10 November, 2004 (Wintoft et al., 2005) is characterized by the three largest magnetic storms (SYM-H $<-220 \mathrm{nT})$ for which the CLF and HER H-component data were severely disturbed (Fig. 8(b) right). Dashed lines indicate the onset of the principal geomagnetic events. At the onset of the first storm, which started around 21:55 UT, the spectral exponents feature a rapid change from a smaller to a larger value at 22:00 UT, clearly illustrated by the transition T1 at the CLF and HER observatories. Looking at the details, we note that this transition coincides with the first part of the main phase, followed by some fluctuations with an average value $\beta(t)>2$ until the end of November 8 .

On 9 November at the CLF observatory, the scaling exponent increases gradually from an antipersistent to persistent value immediately after the storm onset around 21:00, as illustrated in Fig. 8(b) right, correlated with the main phase of the storm. However, at the HER observatory, the spectral exponent fluctuates around an average value of $\beta(t) \approx 2$.

Again, during the third storm on November 10 (around 10:00 UT), we note that the exponents gradually increase, reaching a maximum value of $\beta(t) \approx 3$ correlated to the main phase.

In the case of the THL observatory, the behaviour of the $\beta(t)$ exponent curve is not exactly similar to that of
CLF and HER, even though the beginning/end of the storm are also well indicated by phase transitions T1 for May 97, T1-T4 for July 2000, T1-T2 for October 2003, and T1 for 4 November, 2003, as illustrated by Fig. 9. All these transitions of scaling exponents from smaller to larger values are correlated with intervals containing intense (and smaller) space storms.

However, the scaling exponent shows no increasing values at the storm onset for the remaining events (September 2002 and November 2003) of Fig. 9, and it fluctuates with average values $2<\beta(t)<3$ close to the geomagnetic events.

\section{Conclusions}

By monitoring the temporal evolution of the fractal spectral characteristics in the geomagnetic time series, we have found that distinct changes in the associated scaling exponents indicate a transition from a normal low-activity state to an unusual/high-activity state, related to intense magnetic storms. The transition is accompanied by a particular signature of a rapid change in the temporal scaling of fluctuations in a storm-time geomagnetic time series. This indicates the formation of a new dynamical phase considerably more ordered than the background state. This result is in accordance with the entropy decrease shown by Balasis et al. (2008, 2009). A transition from a random to a correlated state was actually observed and discussed during the active periods of storm in the Dst index (Balasis et al., 2006) and the SYM-H index (Wanliss, 2005a; Wanliss and Dobias, 2007).

To emphasize the detailed analysis of the scaling exponent behaviour related to the six intense storms of cycle 23, we have focused our results mainly over 3- and 4-day intervals, comprising each storm. Therefore, the statistical properties of the long-range correlations described by the temporal evolution of the scaling exponents are located around these disturbance periods. This indicates the transition from antipersistent to persistent statistics around the beginning of the main phase. This transition is more prominently seen at mid-latitudes (CLF and HER observatories) than at high latitudes (THL observatory). This can be attributed to the presence of auroral currents that influence geomagnetic variations at high latitudes.

Other studies also indicate the existence of two different regimes in the dynamics of the magnetosphere. Sitnov et al. (2001) suggested that substorm dynamics resembles second-order phase transitions, while magnetic storms reveal features of first-order non-equilibrium transitions. The antipersistency/persistency meet well the second-order/first-order phase transition, correspondingly. The shape of the scaling exponent curves in Figs. 6, 7 and 8 features the dynamics of the time series complexity close to its instability. This transition is indicated by the change from $\beta(t)<2$ prior to the storm onset and $\beta(t)>2$ for some time after the onset. These observations are consistent with conclusions obtained by Alava (2003), Chang et al. (2003, 2004), Balasis et al. (2006), and Wanliss and Dobias (2007). The obvious presence of fractal scaling behaviour at all times, and the variation in scaling exponents from quiet to active intervals, suggest that the non-equilibrium mag- 

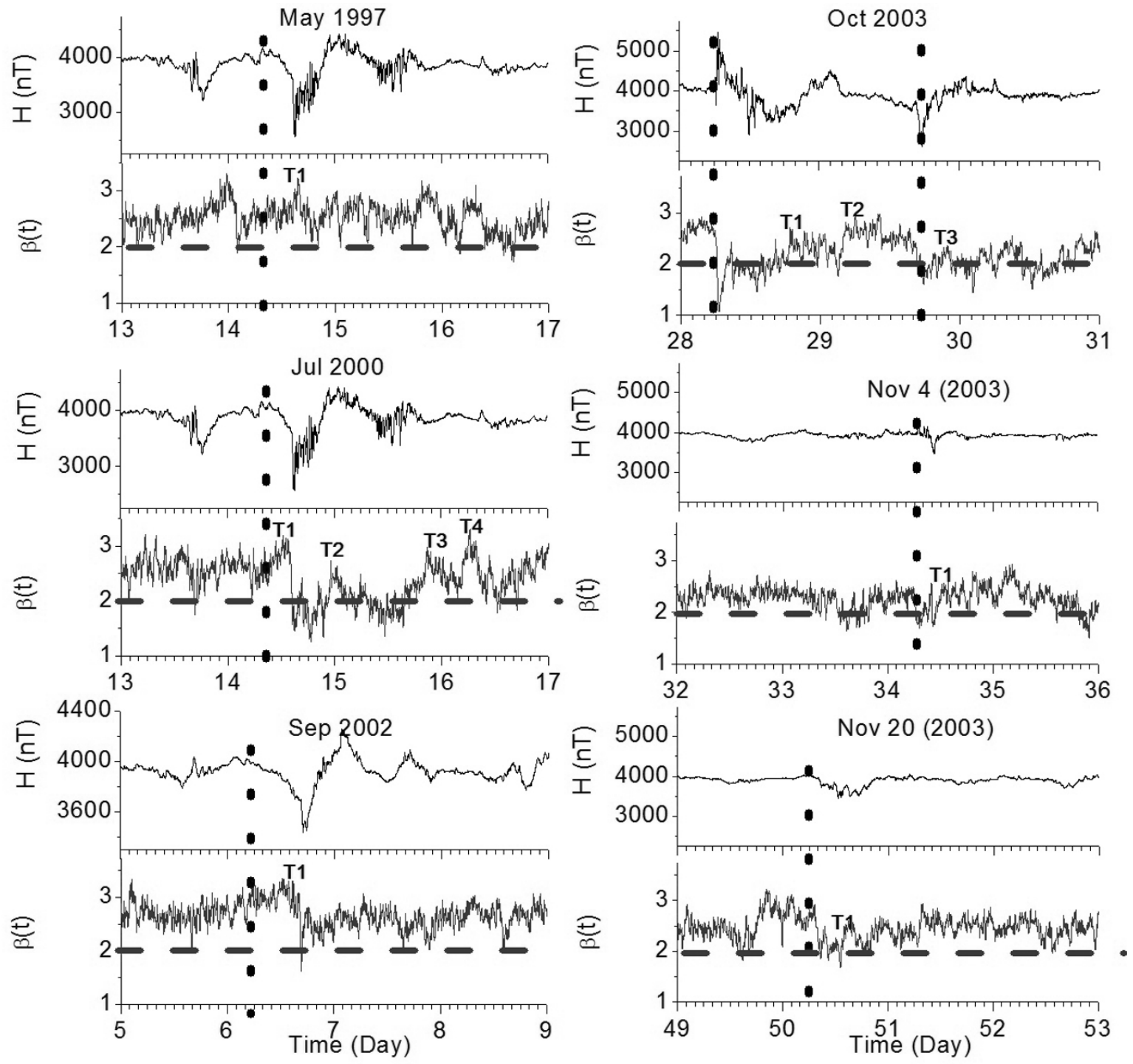

Fig. 9. Horizontal component temporal behaviour, as seen by one-minute values at the THL observatory, and associated wavelet spectral exponents. From top-left (1st column) to bottom-right (2nd column) are shown in chronological order the events of 15 May, 1997, 15 July, 2000,7 September, 2002, October, 2003, 4 November, 2003 and 20 November, 2003.

netosphere system, represented by time series fluctuations, exists in a critical configuration.

Whilst prior to the onset of a storm the nonlinear scaling exponent usually decreases in antipersistent behaviour with time, storm onset results in a remarkable change with the emergence of persistent properties. Transition to a more ordered state, as the magnetic storm peak approaches, results in a higher-scaling exponent, indicating a higher organization, or lower entropy, of the Earth's magnetosphere system around magnetic storms. The emergence of longrange correlations, i.e. the appearance of memory effects, implies a multi-time-scale communal activity of numerous activated units. Following Chang (1999) and Consolini and Chang (2001, 2002), these observations suggest that under the influence of the solar wind, the magnetosphere can be considered to be in a globally non-equilibrium critical state.

Finally, this study indicates that the dynamics in the magnetosphere can be characterised in more detail when highresolution data are used and more objective mathematical tools are applied. The estimation of the persistence charac- ter of the magnetic field fluctuations linked to the magnetic storms can be proposed as an indicator for forecasting magnetic activity. This is an ongoing study to assess the robustness of the approach by considering a larger raw dataset.

Acknowledgments. We thank magnetic observatories and national agencies for their efforts in producing and archiving magnetic data. J. A. Wanliss and an anonymous reviewer, are greatly acknowledged for their thorough reviews which improve the quality of the manuscript. The contribution of Makoto Uyeshima is also warmly acknowledged.

\section{References}

Alava, M., Self-organized criticality as a phase transition, in Advances in Condensed Matter and Statistical Mechanics, edited by E. Korutcheva and R. Cuerno, Nova Science Publishers, 2003.

Alex, S., S. Mukherjee, and B. M. Pathan, Multiple flare occurrences and the geomagnetic storm characteristics during solar cycle 23 ILWS workshop 2006, Goa, February 19-24, 2006.

Angelopoulos, V., J. P. McFadden, D. Larson, C. W. Carlson, S. B. Mende, H. Frey, T. Phan, D. G. Dibeck, K.-H. Glassmeier, U. Auster, E. Donovan, I. R. Mann, J. Rae, C. T. Russell, A. Runov, X.-Z Zhou, and L. Kepko, Tail reconnection, triggering substorm onset, Science, 321, 931- 
935, doi:10.1126/science.1160495, 2008.

Baker, D. N., Efforts of the Sun on the Earth's environment, J. Atmos. Sol. Terr. Phys., 62, 1669-1681, 2000.

Balasis, G., I. A. Daglis, P. Kapiris, M. Mandea, D. Vassiliadis, and K. Eftaxias, From pre-storm to magnetic storms: a transition described in terms of fractal dynamics, Ann. Geophys., 24, 3557-3567, 2006.

Balasis, G., I. A. Daglis, C. Papadimitriou, M. Kalimeri, A Anastasiadis, and K. Eftaxias, Dynamical complexity in Dst time series using non-extensive Tsallis entropy, Geophys. Res. Lett., 35, L14102, doi:10.1029/2008GL034743, 2008. (see AGU Editor's Choice: Space Weatherhttp://www.agu.org/pubs/journals/virtual/editors_choice/si.shtml).

Balasis, G., I. A. Daglis, C. Papadimitriou, M. Kalimeri, A. Anastasiadis, and $\mathrm{K}$. Eftaxias, Investigating dynamical complexity in the magnetosphere using various entropy measures, J. Geophys. Res., 114, A00D06, doi:10.1029/2008JA014035, 2009.

Balasis, G., I. A. Daglis, A. Anastasiadis, and K. Eftaxias, Detection of dynamical complexity changes in Dst time series using entropy concepts and rescaled range analysis, in The Dynamic Magnetosphere, edited by W. Liu, and M. Fujimoto, IAGA Special Sopron Book Series, Springer, vol. 3, part 3, 211-220, doi:10.1007/978-94-007-0501-2_12, 2011.

Balasis, G., I. A. Daglis, E. Zesta, C. Papadimitriou, M. Georgiou, R. Haagmans, and K. Tsinganos, ULF wave activity during the 2003 Halloween superstorm: multipoint observations from CHAMP, Cluster and Geotail missions, Ann. Geophys., 30, 1751-1768, doi:10.5194/angeo30-1751-2012, 2012

Bothmer, V. and R. Schwenn, Signatures of Fast CMEs In Interplanetary Space, Adv. Space Res., 17, 319-322, 1995.

Chang, T., Self-organized criticality, multi-fractal spectra, sporadic localized reconnections and intermittent turbulence in magnetotail, Phys. Plasmas, 6, 4137-4145, 1999.

Chang, T., S. W. Y Tam, C. C Wu, and G. Consolini, Complexity, Forced and/or Self-organized criticality, and topological phase transitions in space plasmas, Space Sci. Rev., 107, 425-445, 2003.

Chang, T., S. W. Y. Tam, and C. C. Wu, Complexity Induced Anisotropic Bimodal Intermittent Turbulence in Space Plasma, Phys. Plasmas, 11, 1287-1299, 2004.

Consolini, G. and T. Chang, Magnetic field topology and criticality in geotail dynamics: relevance to substorm phenomena, Space Sci. Rev., 95, 309-321, 2001.

Consolini, G. and T. Chang, Complexity, magnetic field topology, criticality, and metastability in magnetotail dynamics, J. Atmos. Sol. Terr Phys., 64, 541-549, 2002.

Daglis, I. A., D. N. Baker, Y. Galperin, J. G. Kappenman, and L. J. Lanzerotti, Technological impacts of space storms: Outstanding issues, Eo Trans. AGU, 82, 585-585, doi:10.1029/01EO00340, 2001.

Daubechies, I., Ten Lectures on Wavelets, 357 pp., SIAM Press, Philadelphia, 1992.

Farrugia, C. J., H. Matsui, H. Kucharek, R. B. Torbert, C. W. Smith, V. K Jordanova, K. W. Ogilvie, R. P. Lepping, D. B. Berdichevsky, T. Terasawa, J. Kasper, T. Mukai, Y. Saito, and R. Skoug, Interplanetary coronal mass ejection and ambient interplanetary magnetic field correlations during the Sun-Earth connection events of October-November 2003, $J$ Geophys. Res., 110, 1-16, 2003.

Freeman, J. W., Storm in Space, 139 pp, Cambridge University Press, UK, 2001.

Gaci, S., N. Zaourar, M. Hamoudi, and M. Holschneider, Local regularity analysis of strata heterogeneities from sonic logs, Nonlin. Processes Geophys., 17, 1-12, 2010.

Gonzalez, W. D., J. A. Joselyn, Y. Kamide, H. W. Kroehl, G. Rostoker, B. T. Tsurutani, and V. M. Vasyliunas, What is a geomagnetic storm?, $J$ Geophys. Res., 99, 5771-5792, 1994.

Gopalswamy, N., L. Barbieri, G. Lu, S. P. Plunkett, and R. M. Skoug, Introduction to the special section: Violent Sun-Earth connection events of October-November 2003, Geophys. Res. Lett., 32, doi: 10.1029/2005GL022348, 2005

Gosling, J. T., S. J. Bame, D. J. McComas, and J. L. Phillips, Coronal mass ejections and large geomagnetic storms, Geophys. Res. Lett., 17, 901-904, 1990

Grossmann, A. and J. Morlet, Decomposition of hardy functions into square integrable wavelets of constant shape, SIAM J. Math. Anal., 15, 723-736, 1984.

Heneghan, C. and G. McDarby, Establishing the relation between detrended fluctuation analysis and power spectral density analysis for stochastic processes, Phys. Rev. E., 62, 6103-6110, 2000.

Holschneider, M., Wavelets: an Analysis Tool, Clarendon, Oxford, Eng- land, 1995.

Holschneider, M., M. S. Diallo, M. Kulesh, M. Ohrnberger, E. Lück, and F. Scherbaum, Characterization of dispersive surface waves using continuous wavelet transforms, Geophy. J. Int., 163, 463-478, 2005.

Ivanov, V. V., N. M Rotanova, and E. V. Kovalevskaya, The Wavelet Analysis as applied to the Study of Geomagnetic Disturbances, Geomagn. Aeron., 41, 583-591, 2001.

Iyemori, T., Storm time magnetospheric currents inferred from midlatitude geomagnetic field variations, J. Geomag. Geoelectr., 42, 1249-1265, 1990.

Kamide, Y., W. Baumjohann, I. A. Daglis, W. D. Gonzalez, M. Grande, J. A. Joselyn, R. L. McPherron, J. L. Phillips, E. G. D. Reeves, G. Rostoker, A. S. Sharma, H. J. Singer, B. T. Tsurutani, and V. M. Vasyliunas, Current understanding of magnetic storms: Storm-substorm relationships, J. Geophys. Res., 103, 17705-17728, 1998.

Kantelhardt, J. W., Fractal and Multifractal Time Series, in Springer encyclopaedia of complexity and system science, Springer, arXiv: 0804.074v1 [Physics.data-an], 2008.

Kantelhardt, J. W., E. Koscielny-Bunde, D. Rybski, P. Braun, A Bunde, and S. Havlin, Long-term persistence and multifractality of precipitation and river runoff records, J. Geophys. Res., 111, doi: 10.1029/2005JD005881, 2006

Kovacs, P., V. Carbone, and Z. Vörös, Wavelet based filtering events from geomagnetic time-series, Planet. Space Sci., 49, 1219-1231, 2001.

Lui, A. T. Y., Multiscale phenomena in the near-Earth magnetosphere, $J$ Atmos. Sol.-Terr. Phys., 64, 125-143, 2002.

Malamud, B. D. and D. L. Turcotte, Self-affine time series: measures of weak and strong persistence, J. Stat. Plan. Inf., 80, 17-196, 1999.

Mallat, S., Une Exploration des Signaux en Ondelettes, Edition de 1'Ecole polytechnique, $636 \mathrm{pp}, 2000$

Mandea, M. and G. Balasis, The SGR 1806-20 magnetar signature on the Earth's magnetic field, Geophys. J. Int., 167, 586-591, doi:10.1111/j.1365-246X.2006.03125.x, 2006.

Mandelbrot, B. B., Self-affine fractals and fractal dimension, Phys. Scripta, 32, 257-260, 1985.

Mandelbrot, B. B. and J. W. Ness, Fractional Brownian motions, fractiona noises and applications, SIAM Rev., 10, 422-437, 1968.

Mendes, O. J., M. D. Oliveira, A. Mendes da Costa, and A. L. Clùa de Gonzalez, Wavelet analysis applied to magnetograms: Singularity detections related to geomagnetic storms, J. Atmos. Sol. Terr. Phys., 67, 1827-1836, 2005.

Meyer, Y., Wavelets: Algorithms \& Applications, 135 pp, SIAM Press, Philadelphia, 1993.

Pilkington, M. and J. P. Todoeschuck, Stochastic inversion for scaling geology, Geophys. J. Int., 102, 205-217, 1990.

Pilkington, M. and J. P. Todoeschuck, Naturally smooth inversion with a priori information from well logs, Geophysics, 56(11), 1811-1818, 1991.

Richardson, I. G. and J. Zhang, Multiple-step geomagnetic storms and their interplanetary drivers, Geophys. Res. Lett., 35, L06S07, doi:10.1029/2007GL032025, 2008.

Sitnov, M. I., A. S. Sharma, K. Papadopoulos, and D. Vassiliadis, Modeling substorm dynamics of the magnetosphere: From self-organization and self-organized criticality to nonequilibrium phase transitions, Phys. Rev. E, 65, doi:10.1103/PhysRevE.65.016116, 2001.

Torrésani, B., Analyse Continue Par Ondelettes, Inter Editions/CNRS Edition, $239 \mathrm{pp}, 1995$.

Tsurutani, B. T. and W. D Gonzalez, The future of geomagnetic storm predictions: implications from recent solar and interplanetary observations, J. Atmos. Sol. Terr. Phys., 57, 1369-1384, 1995.

Turcotte, D. L., Fractal and Chaos in Geology and Geophysics, 398 pp, Cambridge Univ. Press, 1997.

Uritsky, V., A. Klimas, and D. Vassiliadis, Comparative study of dynamical critical scaling in the auroral electrojet index versus solar wind fluctuations, Geophys. Res. Lett., 28, 3809-3812, 2001.

Uritsky, V. M., A. J. Klimas, and D. Vassiliadis, Critical finite-size scaling of energy and lifetime probability distributions of auroral emissions, Geophys. Res. Lett., 33, L08102, doi:10.1029/2005GL025330, 2006.

Wanliss, J., Fractal properties of SYM-H during quiet and active times, $J$. Geophys. Res., 110, doi:10.1029/2004JA010544, 2005a.

Wanliss, J. A., Statistical precursors to space storm onset, in Multiscale Coupling of Sun-Earth Processes, edited by A. T. Y. Lui, Y. Kamide and G. Consolini, , Elsevier, 2005b.

Wanliss, J. A. and P. Dobias, Space storm as a dynamic phase transition, $J$. Atmos. Sol. Terr. Phys., 69, 675-684, 2007.

Wanliss, J. and K. M. Showalter, High-resolution global storm in- 
dex: Dst versus SYM-H, J. Geophys. Res., 111, A02202, doi:10.1029/2005JA011034, 2006.

Wintoft, P., M. Wik, H. Lundstedt, and L. Eliasson, Predictions of local ground geomagnetic field fluctuations during the 7-10 November 2004 events studied with solar wind driven models, Ann. Geophys., 23, 1-7, 2005.

Zaourar, N., M. Hamoudi, and L. Briqueu, Détection des transitions lithologiques de la composante fractale des diagraphies par Transformée en Ondelettes Continue, C. R. Geosc., 338, 514-520, 2006.

Zaourar, N., R. Mebarki, M. C. Berguig, M. Hamoudi, and M. Parrot, Modelling the stochastic component of seismo-electromagnetic Time series recorded by Demeter, Proceedings of the 2nd International Symposium on Radio Systems and Space Plasma, ISRSSP, Sofia, Bulgaria, 25-27 August, 2010.

Zhang, J., J. Woch, S. K. Solanki, R. von Steiger, and R. Forsyth, Interplanetary and solar surface properties of coronal holes observed during solar maximum, J. Geophys. Res., 108, doi:10.1029/2002JA009538, 2003.

N. Zaourar, M. Hamoudi (e-mail: hamoudi@gfz-potsdam.de), M. Mandea, G. Balasis, and M. Holschneider 